・研究报告・

\title{
中国入侵克隆植物入侵性、克隆方式及地理起源
}

\author{
王 宁* 李卫芳 周 兵 间小红 \\ (井冈山大学生命科学学院, 江西吉安 343009)
}

\begin{abstract}
摘要: 通过查阅和整理相关文献资料, 分析了中国入侵克隆植物的入侵性、克隆方式及地理起源等。目前, 在中 国的515种入侵植物中, 克隆植物至少有 196 种, 占总数的 $38.1 \%$ 。这些入侵克隆植物隶属 46 科, 其中禾本科、菊科 和豆科植物占多数, 以人为有意引入为主。在入侵危害等级1-5中, 属于第 5 等级(有待观察类)的克隆植物占多数。 卡方分析表明, 中国入侵植物的危害等级与其是否具有克隆性关系不显著, 但显著依赖于其是否具有根状茎。多 年生和1-2年生草本植物构成中国入侵克隆植物的主体, 分臬型、匍匐茎型和根茎型等繁殖方式在草本类入侵植物 中较为常见。多数入侵克隆植物地理起源上来自美洲地区。这些结果表明, 在我国今后引种活动中需要格外警惕 根状茎型克隆植物以及美洲起源的克隆植物。此外, 由于现有文献资料有待完善, 入侵克隆植物的数量和比例可 能被低估。因此, 在今后工作中应加强入侵植物的野外生长观测, 进而确切判定中国入侵克隆植物的数量和比例。 关键词: 入侵植物; 克隆性; 克隆繁殖; 生长型; 地理起源
\end{abstract}

\section{Invasiveness, clonal form and geographical origin of invasive clonal plant species in China}

\author{
Ning Wang*, Weifang Li, Bing Zhou, Xiaohong Yan \\ School of Life Sciences, Jinggangshan University, Ji'an, Jiangxi 343009
}

\begin{abstract}
The invasiveness, clonal form and geographical origin of invasive clonal plant species in China were analyzed based on some literature reviews. Of the 515 invasive plant species in China, at least 196 (38.1\%) were clonal plant species. These invasive clonal plant species belong to 46 families, and most of them are from Gramineae, Asteraceae and Leguminosae. The majority of the invasive clonal plant species were introduced intentionally by human activity. In the 1-5 invasiveness ranks, most invasive clonal plant species belong to the rank 5 (i.e. species requiring further observation). Chi-square tests showed that species invasiveness ranks did not significantly depend on the presence/absence of clonality, but that they significantly depended on the presence/absence of rhizomes. Perennial and annual herbs accounted for the majority of the 196 invasive clonal plant species. Many invasive clonal herbs reproduce asexually by tillers, stolons or rhizomes. The primary geographical origin of these invasive clonal plant species was America. The results suggest that in future attention should be paid when rhizomatous clonal plant species or clonal plant species from America are introduced. However, our methods may have underestimated the number and proportion of invasive clonal plant species because of limited information about modes of reproduction in the literature. Thus, fieldwork should be conducted to check the clonality of each species to accurately analyze the number and proportion of invasive clonal plant species in China in future.
\end{abstract}

Key words: invasive plant species; clonality; clonal reproduction; growth form; geographical origin

外来植物入侵是指在自然状态或人类作用下, 植物种类在异地获得生长与繁殖的现象 (彭少麟和 向言词, 1999; Richardson et al, 2000)。近几十年来,
受全球变化及国际贸易自由化等多种因素的影响, 植物入侵已对入侵地的生物多样性、生态环境及经 济造成了严重危害 (Pimentel et al，2000，2001;

收稿日期: 2015-07-02; 接受日期: 2015-11-17

基金项目: 国家自然科学基金(31240015 和 31360090)和江西省自然科学基金(20151BAB204011)

* 通讯作者 Author for correspondence. E-mail: wangning13@126.com 
Richardson et al, 2000; Callaway \& Maron, 2006; 间 小玲等, 2012, 2014)。以往研究发现, 许多恶性入侵 植物是典型的克隆植物, 其多样化的繁殖方式和适 应机制增强了其环境适应性和入侵危害性(Pyšek et al, 2003; Liu et al, 2006; 王宁, 2011; Roiloa et al, 2014)。例如, 空心莲子草(Alternanthera philoxeroides)不仅可以通过断裂的匍匐茎在水陆两种生境 中进行繁殖, 而且还可以通过根状茎、宿根等多种 方式进行繁殖(Pan et al, 2006; Wang et al, 2009; Dong et al, 2012)。因此, 加强外来克隆植物入侵性 的研究已成为许多生态学家的共识(董鸣等, 2007; 刘建等, 2010; 董鸣, 2011; Roiloa et al, 2014)。

对一些入侵克隆植物来说, 克隆性在个体、种 群或群落水平上能显著影响其入侵危害性的表达 (Wang et al, 2008; Yu et al, 2009; 董鸣, 2011)。然而 这种显著性影响是否具有普遍性? 具有克隆生长 特性的入侵植物, 其入侵性是否较一般入侵植物更 强? 相关研究者一直对此进行积极探索, 但结论并 不一致。例如, Liu等(2006)研究了中国的126种入侵 植物, 发现其入侵性与克隆性之间存在显著相关 性; Song等(2013a)发现克隆植物的克隆整合能力与 其全球入侵性存在正相关。然而, 也有其他一些研 究认为外来植物的入侵性与克隆性并不存在相关 关系(王宁, 2010, 2011; Song et al, 2013b; 肖望君等, 2014)。例如, 王宁(2011)对美国北卡罗来纳州的112 种入侵植物进行了分析, 未发现其入侵性与克隆性 显著相关; Song等(2013b)研究了39种匍匐茎型克隆 植物, 发现其单节克隆片段(即具有一个节的克隆 片段) 克隆更新能力与其全球入侵性不存在显著相 关性。

由于中国入侵植物早期全国性本底调查工作 开展有限, Liu等(2006)的研究中仅包含了中国126 种入侵植物。事实上, 多数入侵植物于 20 世纪 90 年 代以前就入侵至中国, 但系统性资料整理分析只是 从近二十年才开始, 而不同研究者对入侵植物种类 及其入侵性的评价标准也存在差异, 这无疑影响了 人们对克隆植物入侵性的认识。近十几年来, 随着 入侵植物本底调查资料和评价体系的不断完善, 被 确认的中国入侵植物数量大幅增加, 研究者目前已 归纳整理出隶属于5个入侵危害等级的515种中国 入侵植物(马金双, 2013; 间小玲等, 2014)。因此, 有 必要重新审视中国入侵克隆植物的入侵性、克隆方
式及地理起源, 这可能会提高人们对克隆植物入侵 性的认识, 从而不断完善相应的防控措施。

\section{1 方法}

\section{1 数据来源}

截至目前, 中国被确定有515种入侵植物(马金 双, 2013; 间小玲等, 2014)。通过查询和利用相关文 献资料(Liu et al, 2006; 万方浩等, 2009, 2012; 徐海 根和强胜, 2011)和一些著名的互联网搜索平台(如 谷歌(www.google.com)、维基百科(www.wikipedia. org)和百度(www.baidu.com)), 以及国内外部分文 献资料数据库, 如Web of Science (thomsonreuters. com)、中国知网(CNKI, www.cnki.net)、美国农业部 (USDA) Fact Sheets \& Plant Guides数据库(plants. usda.gov/java/factSheet)、国际应用生物科学中心 (CABI) Invasive Species Compendium数据库(www. cabi.org/isc)、中国植物志数据库(FRPS, frps.eflora. cn)、全球入侵生物数据库(GISD, www.issg.org/ database/welcome)、CLO-PLA3数据库(clopla.butbn. cas.cz)、Pacific Island Ecosystems at Risk数据库 (PIER, www.hear.org/pier/index.html)、eFloras数据库 (www.efloras.org)收录下的Flora of China和Flora of North America、PlantNET 数据库 (plantnet.rbgsyd. nsw.gov.au)、The Pacific Bulb Society数据库(PBS, www.pacificbulbsociety.org)、AgroAtlas数据库(www. agroatlas.ru/en/about/index.html)、美国宾州大学Penn State Extension数据库(extension.psu.edu)、美国Illinois Wildflowers植物数据库(www.illinoiswildflowers. info)、美国Ohio Perennial \& Biennial Weed Guide植 物数据库 (www.oardc.ohio-state.edu/weedguide/listall.asp)、印度 Kerala Plants 植物数据库 (keralaplants.in/index.html)、中国外来入侵植物数据库 (www.agripests.cn/shownews.asp?news_id=26), 对上 述515种入侵植物的具体繁殖方式进行文献资料搜 索确认。排除了目前文献记载中的那些仅依靠种子 繁殖或人为处理(如扦插、压条和分根等)后才可无 性繁殖的植物种类, 将其中记载为具备自然克隆繁 殖方式的植物标定为入侵克隆植物, 最终归纳整理 出中国入侵克隆植物名录(附录1)。

\section{2 统计分析}

统计分析了中国入侵克隆植物的入侵性、克隆 方式、生长型及地理起源等指标。马金双(2013)和 
间小玲等(2014)根据生物学特性和生态学特性、原 产地自然地理分布信息、入侵范围、对生态环境的 危害和对国民经济产生的影响等, 将515种中国入 侵植物分为 5 个等级: (1)恶性入侵类; (2)严重入侵 类; (3)局部入侵类; (4)一般入侵类和(5)有待观察 类。基于上述5级划分标准, 本研究使用SPSS 22.0 统计软件中的卡方检验法对 515 种中国入侵植物的 入侵等级与其是否具有克隆性之间的关系进行了 分析。引入路径分为有意引进和无意引进; 克隆繁 殖方式分为: 匍匐茎型、根状茎型、珠芽型、分箵 型、块茎型、球茎型、鳞茎型、根出条型、根䢃裂 型、块根型和宿根型等(宋明华, 2002; 于飞海, 2002; 董鸣, 2011); 生长型分为: 乔木、灌木、藤本、多年 生草本植物、1-2年生草本植物、水生草本植物等; 地理起源分为: 亚洲、非洲、欧洲、美洲和大洋洲。

\section{2 结果}

\section{1 所属科和引入方式}

中国入侵克隆植物总计 196 种(附录1), 占入侵 植物总数的 $38.1 \%$, 隶属 46 个科(图 1$)$ 。种数较多的 是禾本科、菊科和豆科植物, 分别有 58 种、 27 种和 15 种, 3 科共计 100 种, 占入侵克隆植物的 $51.0 \%$; 其 次为旋花科、柳叶菜科、石蒜科、仙人掌科和玄参

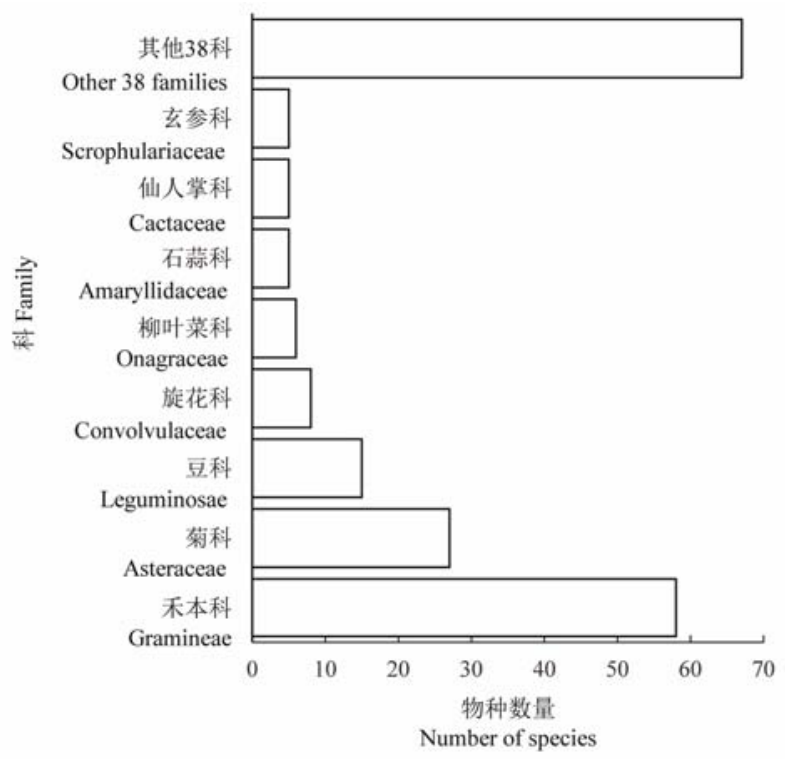

图1入侵克隆植物所属科的物种数量分布

Fig. 1 Number of invasive clonal plant species in different families
科, 分别有 8 种、 6 种、 5 种、 5 种和 5 种， 5 科共计 29 种, 占入侵克隆植物的 $14.8 \%$; 剩余 67 种入侵克隆 植物散布于另外 38 个科中, 占总种数的 $34.2 \%$ 。在引 入路径上, 155 种入侵克隆植物是人为引入, 占总种 数的 $79.1 \%$ 。

\section{2 入侵性和克隆方式}

表1显示, 各等级入侵克隆植物种数随入侵性 增强呈下降趋势, 但占相应等级入侵植物总数的比 例差别不大(34.8-47.1\%)。克隆繁殖方式为根状茎、 匍匐茎、分菜和其他类型 (如球茎、鳞茎、块茎、块 根、根蓝等)的入侵植物种数分别为 $50 、 31 、 45$ 和 111 , 分别占入侵克隆植物种数的 $25.5 \% 、 15.8 \% 、 23.0 \%$ 和 $56.6 \%$ 。对 515 种中国入侵植物的入侵性与其是否 存在克隆性进行卡方检验, 发现外来植物入侵危害 等级与其克隆性有无 $(P=0.72)$ 、匍匐茎有无 $(P=$ $0.14)$ 和分䔯有无 $(P=0.83)$ 之间均不存在显著的相 关性，但显著依赖于这些植物是否具有根状茎繁殖 这一特征 $(P<0.01)$ 。根状茎植物的比例, 在入侵危 害最严重的等级(等级1)中(20.6\%)显著高于其他危 害等级(1.4-16.3\%; 表1)。

\section{3 生长型和克隆方式}

表2显示, 196种入侵克隆植物中, 多年生草本 植物种数占绝对优势, 1-2年生草本植物次之, 水生 草本、藤本和灌木植物相对较少, 乔木种数最少。 在克隆繁殖方式上，1-2年生草本植物中分藥型种 数较多, 多年生草本植物中根状茎型和分菜型种数 居多，而其他生长型的入侵克隆植物并不以上述3

\section{表1 不同入侵性等级和克隆方式下中国入侵植物种数}

Table 1 Number of invasive plant species in China with different invasiveness ranks and clonal forms

\begin{tabular}{|c|c|c|c|c|c|c|}
\hline \multirow{2}{*}{$\begin{array}{l}\text { 等级 } \\
\text { Rank }\end{array}$} & \multirow{2}{*}{$\begin{array}{l}\text { 入侵植物种数 } \\
\text { No. of invasive } \\
\text { plants }\end{array}$} & \multicolumn{5}{|c|}{ 克隆植物种数 No. of clonal plant species } \\
\hline & & $\begin{array}{l}\text { 总数 } \\
\text { Total }\end{array}$ & $\begin{array}{l}\text { 根状茎 } \\
\text { Rhizome }\end{array}$ & $\begin{array}{l}\text { 匍匐茎 } \\
\text { Stolon }\end{array}$ & $\begin{array}{l}\text { 分睬 } \\
\text { Tiller }\end{array}$ & $\begin{array}{l}\text { 其他 } \\
\text { Others }\end{array}$ \\
\hline 1 & 34 & 16 & 7 & 5 & 3 & 9 \\
\hline 2 & 69 & 24 & 1 & 5 & 7 & 12 \\
\hline 3 & 85 & 33 & 6 & 5 & 7 & 24 \\
\hline 4 & 80 & 33 & 13 & 6 & 9 & 16 \\
\hline 5 & 247 & 90 & 23 & 10 & 19 & 50 \\
\hline 合计 & 515 & 196 & 50 & 31 & 45 & 111 \\
\hline
\end{tabular}

有些植物可能具有多种克隆繁殖器官。

Some plant species may have multiple clonal organs. 
表2 不同生长型和克隆方式下中国入侵克隆植物种数

Table 2 Number of invasive clonal plant species in China with different growth forms and clonal forms

\begin{tabular}{|c|c|c|c|c|c|}
\hline $\begin{array}{l}\text { 生长型 } \\
\text { Growth form }\end{array}$ & 总数 & 根状茎 & 匍匐茎 & 分睬 & 其他 \\
\hline 1-2年生草本 & 39 & 2 & 6 & 18 & 15 \\
\hline Annual or bienı & & & & & \\
\hline $\begin{array}{l}\text { 多年生草本 } \\
\text { Perennial herb }\end{array}$ & 119 & 46 & 19 & 26 & 63 \\
\hline $\begin{array}{l}\text { 水生草本 } \\
\text { Aquatic herb }\end{array}$ & 14 & 1 & 4 & 1 & 10 \\
\hline 藤本 Vine & 10 & 1 & 2 & 0 & 9 \\
\hline 灌木 Shrub & 10 & 0 & 0 & 0 & 10 \\
\hline 乔木 Tree & 4 & 0 & 0 & 0 & 4 \\
\hline 合计 Total & 196 & 50 & 31 & 45 & 111 \\
\hline
\end{tabular}

有些植物可能具有多种克隆繁殖器官。

Some plant species may have multiple clonal organs.

表3 不同入侵性等级和地理起源下中国入侵克隆植物种数

Table 3 Number of invasive clonal plant species in China with different invasiveness ranks and geographical origins

\begin{tabular}{ccccccc}
\hline $\begin{array}{c}\text { 等级 } \\
\text { Rank }\end{array}$ & $\begin{array}{c}\text { 美洲 } \\
\text { America }\end{array}$ & $\begin{array}{c}\text { 欧洲 } \\
\text { Europe }\end{array}$ & $\begin{array}{c}\text { 亚洲 } \\
\text { Asia }\end{array}$ & $\begin{array}{c}\text { 非洲 } \\
\text { Africa }\end{array}$ & $\begin{array}{c}\text { 大洋洲 } \\
\text { Australia }\end{array}$ & $\begin{array}{c}\text { 不详 } \\
\text { Unclear }\end{array}$ \\
\hline 1 & 14 & 2 & 1 & 0 & 0 & 0 \\
2 & 15 & 7 & 2 & 4 & 0 & 0 \\
3 & 18 & 6 & 2 & 7 & 1 & 0 \\
4 & 11 & 16 & 10 & 5 & 0 & 1 \\
5 & 50 & 24 & 14 & 17 & 1 & 1 \\
合计 Total & 108 & 55 & 29 & 33 & 2 & 2 \\
\hline
\end{tabular}

有些植物可能起源于多个洲。

Some plant species may originate from several continents.

种无性繁殖方式为主，而是散布于根葟、块茎和鳞 茎等其他无性繁殖方式中(附录1)。

\section{4 地理起源}

表3显示, 196种入侵克隆植物中, 源自美洲的 种数最多, 其次为欧洲、非洲、亚洲和大洋洲, 仅 有2种植物地理起源不详。对入侵克隆植物来说, 在 每一入侵性等级中, 来自美洲的入侵克隆植物总是 占多数, 特别是在入侵性等级 1 中占绝对优势 (82.4\%)。结合间小玲等(2014)的研究结果, 发现源 自美洲、欧洲、非洲、亚洲和大洋洲的中国入侵植 物中, 克隆植物种数分别占 $34.4 \% 、 56.1 \% 、 52.4 \%$ 、 $34.1 \%$ 和 $15.4 \%$ 。

\section{3 讨论}

在中国入侵克隆植物种类组成中, 禾本科、菊 科和豆科的植物占据多数, 这可能和这些科植物的 自身生长特性密切相关。在这些科中, 禾本科植物 多具有易存活、种子数量多、生长速度快和繁殖方 式多样化等特性(李博等, 2001), 因而很多禾草类入 侵克隆植物能够大量繁殖扩散, 从而造成严重危 害。例如, 互花米草(Spartina alterniflora)不仅可以 通过种子进行传播, 而且凭借其发达的根状茎, 单 株在一年内可繁殖几十甚至上百株(国家环境保护 总局, 2003; 王卿等, 2006)。菊科植物则因具有较高 的观赏价值, 容易被人们在环境绿化时大量引种栽 培。例如，该科的剑叶金鸡菊(Coreopsis lanceolata) 被广泛用于环境绿化后，凭借其高种子结实率、长 期的强分箵能力及短期的游击型克隆生长等特性, 在许多地方已经造成较为严重的危害(曾建军等, 2010, 2012)。豆科植物则是人类食品中淀粉、蛋白 质、油和蔬菜的重要来源之一, 被人们在农业生产 中广泛引种栽培, 而且其生长型比较多样化, 在草 本、灌木和乔木中都有一定数量的种类分布(间小玲 等, 2014)。从引入路径看, 绝大多数入侵克隆植物 是人为有意引入, 这与我国大量引入了具有一定观 赏、食用和药用等价值的外来植物种类有关(王宁 等, 2013; 罗辉等, 2015)。

对入侵植物进行危害等级划分, 有利于采取合 理的防控措施。Liu等(2006)将126种中国入侵植物 按入侵性强弱分为 3 个等级, 并详细说明了划分依 据, 发现中国入侵植物的入侵性与其克隆性密切相 关。而本研究按照马金双(2013)的入侵性5级划分标 准, 对 515种中国入侵植物进行了相关分析, 并未 发现植物入侵性与克隆性之间存在显著相关性。类 似的，以往对江西省和美国北卡罗来纳州、弗吉尼 亚州入侵植物进行的相关分析, 也未发现植物的入 侵性与其克隆性显著相关(王宁，2010，2011; 肖牮 君等, 2014)。这似乎表明选用不同的入侵危害等级 划分标准和入侵植物研究对象可能会得出不同的 研究结论。本研究发现, 根状茎型克隆植物在几种 入侵危害等级中所占比例较高, 这表明根状茎的存 在可能在一定程度上增强了入侵克隆植物的入侵 性。然而, 从入侵植物整体性状来看, 虽然部分恶 
性入侵植物具有强大的无性繁殖能力, 但克隆性对 入侵植物整体入侵性的影响有限。因为在入侵的传 播阶段, 克隆分株往往不能像种子那样有效地进行 远距离传播(Pyšek, 1997)。当然入侵植物的等级划 分并非一成不变, 而是动态并具有时效性的, 随着 研究工作的深入开展, 其入侵级别划分可能会更加 符合客观实际。

在植物生长型方面, 多年生草本植物在中国入 侵克隆植物中占据多数且繁殖方式呈多样化, 这应 该跟其生长特性密切相关。有些多年生入侵克隆草 本植物即使地上部分每年死亡, 但在第二年仍然可 以凭借地下部分的鳞茎、块根和宿根等器官重新萌 发产生新分株; 有些多年生入侵克隆草本植物地下 和地上部分器官均不会当年枯死, 而是保持连续多 年生长(Pan et al, 2006; Wang et al, 2009)。1-2年生入 侵克隆草本植物则以分藓型居多, 这有利于其在局 部生境中快速形成密集的种群竞争优势 (董鸣, 2011)。水生入侵克隆植物则多依靠断枝等方式进行 无性繁殖, 在外力作用下容易产生大量的断裂片段 并迅速形成新分株, 从而加大了治理难度(万方浩 等, 2012)。灌木和乔木类入侵克隆植物的无性繁殖 扩张主要通过断枝、根真等方式进行(徐海根和强 胜, 2011; 侯玉平等, 2015)。

针对上述入侵克隆植物的多样化无性繁殖方 式, 在今后的防控中务必做到全面清除, 以免其残 留植株器官形成二次繁殖入侵。此外需要注意的是, 本研究只统计分析了现有文献资料中记载为具有明 确自然克隆繁殖方式的入侵克隆植物种类, 没有收 录被确认人为处理下(如扞插、压条和分根等)可以进 行无性繁殖的入侵植物种类。在自然界中, 这类植物 也可能存在自然克隆繁殖现象。此外, 早期的很多文 献对一些入侵植物是否存在克隆性没有明确的描述, 尤其是一些克隆性发生现象并不普遍的植物。随着 未来入侵植物野外生长特性研究的不断深入, 可能 会有更多的入侵植物种类被确认为克隆植物。

从地理起源来看, 中国入侵克隆植物多来自美 洲和欧洲, 其次为亚洲和非洲, 大洋洲起源的很少, 这跟中国入侵植物的地理起源分布一致(Liu et al, 2006; 徐海根和强胜, 2011; 闵小玲等, 2014)。在所 有入侵克隆植物中, 美洲起源的占一半以上, 而且 在入侵危害性最强的等级中占绝对优势。例如, 在 2003年国家环境保护总局所公布的中国第一批 16
种外来入侵物种中, 来自美洲的入侵克隆植物就占 了近 $1 / 3$ (国家环境保护总局, 2003)。其主要原因可 能是中国的气候条件整体跟美洲相似(吴晓雯等, 2006; 冯建孟等, 2011)。依据大陆漂移学说, 北美植 物与东亚植物具有相似的遗传背景, 它们可能并未 完全脱化或丧失对原大陆气候的适应能力(强胜和 曹学章, 2000), 这将有利于美洲入侵植物在中国的 成功扩散和定居。此外, 来自欧洲、亚洲和非洲的 入侵克隆植物也占据一定比例, 这可能和中国在历 史上跟上述各洲进行过广泛交流接触有关。例如, 已有研究表明，中国与上述地区内国家间的长期贸 易往来是影响生物入侵危害的重要因素(Callaway et al, 2006; Jenkins \& Mooney, 2006; Weber \& Li, 2008)。

植物入侵性一般被认为是一系列植物功能性 状和环境因子综合作用的结果(刘建等, 2010; Pyšek et al, 2012; van Kleunen et al, 2015)。虽然克隆性在 入侵植物的定居阶段和扩散入侵阶段中具有重要 作用, 能够解释很多入侵克隆植物的入侵性, 但可 能不是判断外来植物能否形成入侵的决定因子, 未 来应加强不同环境因素对植物克隆性与其他功能 性状的综合影响研究。最后需要指出的是, 某些入 侵克隆植物虽然在当前并未表现出较强的入侵性, 但并不能排除其未来爆发的可能性。因此, 在全球 变化和国际贸易日趋增强的背景下，必须加强对外 来入侵克隆植物的长期监管。

致谢: 多位审稿人对本论文提出了诸多修改意见, 在此表示感谢! 同时感谢中国科学院地理科学与资 源研究所宋明华博士和捷克科学院植物研究所Jitka Klimesova博士对入侵克隆植物划分所提出的具体 意见，以及德国Konstanz大学Mark van Kleunen教 授对本论文中数据分析方法的指导。

\section{参考文献}

Callaway RM, Maron JL (2006) What have exotic plant invasions taught us over the past 20 years? Trends in Ecology and Evolution, 21, 369-374.

Callaway RM, Miao SL, Guo Q (2006) Are trans-Pacific invasions the new wave? Biological Invasions, 8, 1435-1437.

Dong BC, Alpert P, Guo W, Yu FH (2012) Effects of fragmentation on the survival and growth of the invasive, clonal plant Alternanthera philoxeroides. Biological Invasions, 14, 1101-1110.

Dong M (2011) Clonal Plant Ecology. Science Press, Beijing. 
(in Chinese) [董鸣 (2011) 克隆植物生态学. 科学出版社, 北京.]

Dong M, Yu FH, An SQ, He WM, Liang SC (2007) Ecological consequences of plant clonality. Chinese Journal of Plant Ecology, 31, 549-551. (in Chinese with English abstract) [董鸣, 于飞海, 安树青, 何维明, 梁士楚 (2007) 植物克 隆性的生态学意义. 植物生态学报, 31, 549-551.]

Feng JM, Dong XD, Xu CD, Zha FS (2011) Risk assessment of alien invasive plants in China and its spatial distribution pattern. Journal of Southwest University (Natural Science Edition), 33(2), 57-63. (in Chinese with English abstract) [冯建孟, 董晓东, 徐成东, 查凤书 (2011) 中国外来入侵 植物的风险评价及空间分布格局. 西南大学学报(自然科 学版), 33(2), 57-63.]

Hou YP, Liu L, Chu H, Ma SJ, Zhao D, Liang RR (2015) Effect of exotic plant Rhus typhina invasion on soil properties in different forest types. Acta Ecologica Sinica, 35, 53245330. (in Chinese with English abstract) [侯玉平, 柳林, 初 航, 马淑杰, 赵丹, 梁荣荣 (2015) 外来植物火炬树(Rhus typhina)入侵对不同林型土壤性质的影响. 生态学报, 35, 5324-5330.]

Jenkins PT, Mooney HA (2006) The United States, China, and invasive species: present status and future prospects. Biological Invasions, 8, 1589-1593.

Li B, Xu BS, Chen JK (2001) Perspectives on general trends of plant invasions with special reference to alien weed flora of Shanghai. Biodiversity Science, 9, 446-457. (in Chinese with English abstract) [李博, 徐炳声, 陈家宽 (2001) 从 上海外来杂草区系剖析植物入侵的一般特征. 生物多样 性, 9, 446-457.]

Liu J, Dong M, Miao SL, Li ZY, Song MH, Wang RQ (2006) Invasive alien plants in China: role of clonality and geographical origin. Biological Invasions, 8, 1461-1470.

Liu J, Li JM, Yu H, He WM, Yu FH, Sang WG, Liu GF, Dong $M$ (2010) The relationship between functional traits and invasiveness of alien plants. Biodiversity Science, 18, 569-576. (in Chinese with English abstract) [刘建, 李钧敏, 余华, 何维明, 于飞海, 桑卫国, 刘国方, 董鸣 (2010) 植物功能性状与外来植物入侵. 生物多样性, 18 , 569-576.]

Luo H, Wang N, Zhao YJ, Zhou B (2015) Species of invasive edible plants in China and their edibility assessment. Chinese Journal of Ecology, 34, 1886-1891. (in Chinese with English abstract) [罗辉, 王宁, 赵雅洁, 周兵 (2015) 中国 可食性入侵植物的种类及其食用价值等级评价. 生态学 杂志, 34, 1886-1891.]

Ma JS (2013) The Checklist of the Chinese Invasive Plants. Higher Education Press, Beijing. (in Chinese) [马金双 (2013) 中国入侵植物名录. 高等教育出版社, 北京.]

Pan XY, Geng YP, Zhang WJ, Li B, Chen JK (2006) Cover shift and morphological plasticity of invasive Alternanthera philoxeroides along a riparian zone in South China. Journal of Plant Ecology (Chinese Version), 30, 835-843. (in Chinese with English abstract) [潘晓云, 耿宇鹏, 张文驹, 李 博, 陈家宽 (2006) 喜旱莲子草沿河岸带不同生境的盖 度变化及形态可塑性. 植物生态学报, 30, 835-843.]

Peng SL, Xiang YC (1999) The invasion of exotic plants and effects of ecosystems. Acta Ecologica Sinica, 19, 560-568. (in Chinese with English abstract) [彭少麟, 向言词 (1999) 植物外来种入侵及其对生态系统的影响. 生态学报, 19 , 560-568.]

Pimentel D, Lach L, Zuniga R, Morrison D (2000) Environmental and economic costs of nonindigenous species in the United States. BioScience, 50, 53-65.

Pimentel D, McNair S, Janecka J, Wightman J, Simmonds C, O’Connell C, Wong E, Russel L, Zern J, Aquino T, Tsomondo T (2001) Economic and environmental thr-eats of alien plant, animal and microbe invasions. Agriculture, Ecosystems and Environment, 84, 1-20.

Pyšek P (1997) Clonality and plant invasion: can a trait make a difference? In: The Ecology and Evolution of Clonal Plants (eds de Kroon H, van Goenendael J), pp. 405-427. Backhuys Publishers, Leidon.

Pyšek P, Brock JH, Bímová K, Mandák B, Jarošík V, Koukolíková I, Pergl J, Štěpánek J (2003) Vegetative regeneration in invasive Reynoutria (Polygonaceae) taxa: the determinant of invasibility at the genotype level. American Journal of Botany, 90, 1487-1495.

Pyšek P, Jarošík V, Hulme PE, Pergl J, Hejda M, Schaffner U, Vilà M (2012) A global assessment of invasive plant impacts on resident species, communities and ecosystems: the interaction of impact measures, invading species' traits and environment. Global Change Biology, 18, 1725-1737.

Qiang S, Cao XZ (2000) Survey and analysis of exotic weeds in China. Journal of Plant Resources and Environment, 9(4), 34-38. (in Chinese with English abstract) [强胜, 曹学章 (2000) 中国异域杂草的考察与分析. 植物资源与环境学 报, 9(4), 34-38.]

Richardson DM, Pyšek P, Rejmánek M, Barbour MG, Panetta FD, West CJ (2000) Naturalization and invasion of alien plants: concepts and definitions. Diversity and Distributions, 6, 93-107.

Roiloa SR, Rodriguez-Echeverria S, Freitas H (2014) Effect of physiological integration in self/non-self genotype $\mathrm{r}$ ecognition in the clonal invader Carpobrotus edulis. Journal of Plant Ecology, 7, 413-418.

Song MH (2002) Distribution of Clonal Plants and Relation of Their Importance to Environment Factors and Species Diversity. $\mathrm{PhD}$ dissertation, Institute of Botany, Chinese Academy of Sciences, Beijing. (in Chinese with English abstract) [宋明华 (2002) 克隆植物的分布及其与环境和生 物多样性的关系. 博士学位论文, 中国科学院植物研究 所, 北京.]

Song YB, Yu FH, Keser HL, Dawson W, Fischer M, Dong M, van Kleunen M (2013a) United we stand, divided we fall: a 
meta-analysis of experiments on clonal integration and its relationship to invasiveness. Oecologia, 171, 317-327.

Song YB, Yu FH, Li JM, Keser LH, Fischer M, Dong M, van Kleunen M (2013b) Plant invasiveness is not linked to the capacity of regeneration from small fragments: an experimental test with 39 stoloniferous species. Biological Invasions, $15,1367-1376$.

State Environmental Protection Administration of China (2003) The List of the First Group of Alien Invasive Species of China. http://www.gov.cn/gongbao/content/2003/content_ 62285.htm (accessed 2015-06-20) [国家环境保护总局 (2003) 中国第一批外来入侵物种名单. http://www.gov. cn/gongbao/content/2003/content_62285.htm（获取日期 2015-06-20)]

van Kleunen, Dawson W, Maurel N (2015) Characteristics of successful alien plants. Molecular Ecology, 24, 1954-1968.

Wan FH, Guo JY, Zhang F (2009) Research on Biological Invasions in China. Science Press, Beijing. (in Chiness) [万方 浩, 郭建英, 张峰 (2009) 中国生物入侵研究. 科学出版 社, 北京.]

Wan FH, Liu QR, Xie M (2012) Biological Invasive: Color Illustrations of Invasive Alien Plants in China. Science Press, Beijing. (in Chiness) [万方浩, 刘全儒, 谢明 (2012) 生物入侵: 中国外来入侵植物图鉴. 科学出版社, 北京.]

Wang N (2010) Study on the invasiveness and clonality of invasive alien plant in Jiangxi Province. Journal of Jinggangshan University (Natural Science Edition), 31(2), 108-112. (in Chinese with English abstract) [王宁 (2010) 江西省外来入侵植物入侵性与克隆性研究. 井冈山大学 学报(自然科学版), 31(2), 108-112.]

Wang N (2011) Invasiveness, clonality and geographic origin of alien invasive plant in North Carolina of America. Journal of Huazhong Agricultural University (Natural Science Edition), 30, 465-469. (in Chinese with English abstract) [王宁 (2011) 美国北卡罗来纳州外来入侵植物入侵性、克隆性 及地理起源研究. 华中农业大学学报(自然科学版), 30, 465-469.]

Wang N, Du L, Zhou B, Yan XH (2013) Species, origins and risk assessment of alien invasive ornamental plants in China. Journal of Huazhong Agricultural University (Natural Science Edition), 32, 28-32. (in Chinese with English abstract) [王宁, 杜丽, 周兵, 间小红 (2013) 中国外来观赏入侵植 物的种类与来源及其风险评价. 华中农业大学学报(自然 科学版), 32, 28-32.]

Wang N, Yu FH, Li PX, He WM, Liu FH, Liu JM, Dong M (2008) Clonal integration affects growth, photosynthetic efficiency and biomass allocation, but not the competitive ability, of the alien invasive Alternanthera philoxeroides under severe stress. Annals of Botany, 101, 671-678.

Wang N, Yu FH, Li PX, He WM, Liu J, Yu GL, Song YB, Dong M (2009) Clonal integration supports the expansion from terrestrial to aquatic environments of the amphibious stoloniferous herb Alternanthera philoxeroides. Plant Biology, 11, 483-489.

Wang Q, An SQ, Ma ZJ, Zhao B, Chen JK, Li B (2006) Invasive Spartina alterniflora: biology, ecology and management. Acta Phytotaxonomica Sinica, 44, 559-588. (in Chinese with English abstract) [王卿, 安树青, 马志军, 赵斌, 陈家宽, 李博 (2006) 入侵植物互花米草一生物学、生 态学及管理. 植物分类学报, 44, 559-588.]

Weber E, Li B (2008) Plant invasions in China: what is to be expected in the wake of economic development? BioScience, 58, 437-444.

Wu XW, Luo J, Chen JK, Li B (2006) Spatial patterns of invasive alien plants in China and its relationship with environmental and anthropological factors. Journal of Plant Ecology (Chinese Version), 30, 576-584. (in Chinese with English abstract) [吴晓雯, 罗晶, 陈家宽, 李博 (2006) 中国外来 入侵植物的分布格局及其与环境因子和人类活动的关系. 植物生态学报, 30, 576-584]

Xiao YJ, Wang N, Zhou B, Yan XH (2014) Invasiveness, clonality and habitat of alien invasive plant in Virginia of America. Journal of Jinggangshan University (Natural Science Edition), 35(1), 103-106. (in Chinese with English abstract) [肖望君, 王宁, 周兵, 间小红 (2014) 美国弗吉尼亚州入 侵植物入侵性、克隆性和分布生境研究. 井冈山大学学报 (自然科学版), 35(1), 103-106.]

Xu HG, Qiang S (2011) Chinese Invasive Alien Species. Science Press, Beijing. (in Chiness) [徐海根, 强胜 (2011) 中 国外来入侵生物. 科学出版社, 北京.]

Yan XL, Liu QR, Shou HY, Zeng XF, Zhang Y, Chen L, Liu Y, Ma HY, Qi SY, Ma JS (2014) The categorization and analysis on the geographic distribution patterns of Chinese alien invasive plants. Biodiversity Science, 22, 667-676. (in Chinese with English abstract) [间小玲, 刘全儒, 寿海洋, 曾宪锋, 张勇, 陈丽, 刘演, 马海英, 齐淑艳, 马金双 (2014) 中国外来入侵植物的等级划分与地理分布格局分 析. 生物多样性, 22, 667-676.]

Yan XL, Shou HY, Ma JS (2012) The problem and status of the alien invasive plants in China. Plant Diversity and Resources, 34, 287-313. (in Chinese with English abstract) [闵 小玲, 寿海洋, 马金双 (2012) 中国外来入侵植物研究现 状及存在的问题. 植物分类与资源学报, 34, 287-313.]

Yu FH (2002) Adaptive Strategies of Clonal Plants Growing in Heterogeneous Environments. PhD dissertation, Institute of Botany, Chinese Academy of Sciences, Beijing. (in Chinese with English abstract) [于飞海 (2002) 克隆植物对异质性 环境的生态适应对策. 博士学位论文, 中国科学院植物 研究所, 北京.]

Yu FH, Wang N, Alpert, P, He WM, Dong M (2009) Physiological integration in an introduced, invasive plant increases its spread into experimental communities and modifies their structure. American Journal of Botany, 96, 1983-1989.

Zeng JJ, Xiao YA, Sun M (2010) Reproductive traits associated 
with invasiveness in Coreopsis lanceolata. Chinese Journal of Plant Ecology, 34, 966-972. (in Chinese with English abstract) [曾建军, 肖宜安, 孙敏 (2010) 入侵植物剑叶金鸡 菊的繁殖特征及其与入侵性之间的关系. 植物生态学报, 34, 966-972.]

Zeng JJ, Xiao YA, Zhou XJ (2012) Clonal reproductive prop- erty and community characteristic of invasive species $\mathrm{Co}$ reopsis lanceolata. Bulletin of Botanical Research, 32, 147-150. (in Chinese with English abstract) [曾建军, 肖宜 安, 周小军 (2012) 入侵植物剑叶金鸡菊克隆繁殖特性 及群落特征. 植物研究, 32, 147-150.]

(责任编委: 于飞海 责任编辑: 黄祥忠)

\section{附录 Supplementary Material}

\section{附录1 中国入侵克隆植物名录}

Appendix 1 Checklist of invasive clonal plant species in China http: //www.biodiversity-science.net/fileup/PDF/2015190-1.pdf 
王宁, 李卫芳, 周兵, 间小红. 中国入侵克隆植物入侵性、克隆方式及地理起源. 生物多样性, 2016, 24 (1), 12-19.

http://www.biodiversity-science.net/CN/10.17520/biods.2015190

\section{附录 1 中国入侵克隆植物名录}

Appendix 1 Checklist of invasive clonal plant species in China

\begin{tabular}{|c|c|c|c|c|c|c|}
\hline $\begin{array}{l}\text { 入侵等级 } \\
\text { Rank }\end{array}$ & $\begin{array}{l}\text { 种 } \\
\text { Species }\end{array}$ & $\begin{array}{l}\text { 科 } \\
\text { Family }\end{array}$ & $\begin{array}{l}\text { 克隆繁殖方式 } \\
\text { Clonal reproduction form }\end{array}$ & $\begin{array}{l}\text { 克隆繁殖方式参考资料 } \\
\text { References of clonal reproduction form }\end{array}$ & $\begin{array}{l}\text { 原产地 } \\
\text { Origin }\end{array}$ & $\begin{array}{l}\text { 引入路径 } \\
\text { Introduction pathway }\end{array}$ \\
\hline$\overline{1}$ & 苼㢣草 Cenchrus echinatus & 禾本科 Gramineae & 分蒕 Tiller & Lü et al, 2011 & 美洲 America & 无意 Unintentionally \\
\hline 1 & 毒麦 Lolium temulentum & 禾本科 Gramineae & 分藍 Tiller & CLO-PLA3 & 欧洲 Europe & 无意 Unintentionally \\
\hline 1 & 石茅 Sorghum halepense & 禾本科 Gramineae & 根状茎 Rhizome & CLO-PLA3 & 欧洲/亚洲 Europe/Asia & 无意 Unintentionally \\
\hline 1 & 互花米草 Spartina alterniflora & 禾本科 Gramineae & 根状茎/分葟 Rhizome/Tiller & Xu \& Qiang, 2011 & 美洲 America & 有意 Intentionally \\
\hline 1 & 紫茎泽兰 Ageratina adenophora & 菊科 Asteraceae & $\begin{array}{l}\text { 根状茎/断枝 } \\
\text { Rhizome/Shoot fragment }\end{array}$ & Xu \& Qiang, 2011 & 美洲 America & 无意 Unintentionally \\
\hline 1 & 白花鬼针草 Bidens alba & 菊科 Asteraceae & 断枝 Shoot fragment & Tian et al, 2010 & 美洲 America & 无意 Unintentionally \\
\hline 1 & 飞机草 Chromalaena odorata & 菊科 Asteraceae & $\begin{array}{l}\text { 根状茎/不定根 } \\
\text { Rhizome/Adventitious root }\end{array}$ & Quan et al, 2011 & 美洲 America & 无意 Unintentionally \\
\hline 1 & 薇甘菊 Mikania micrantha & 菊科 Asteraceae & 匍匐茎 Stolon & Xu \& Qiang, 2011 & 美洲 America & 有意 Intentionally \\
\hline 1 & $\begin{array}{l}\text { 加拿大一枝黄花 } \\
\text { Solidago canadensis }\end{array}$ & 菊科 Asteraceae & $\begin{array}{l}\text { 根状茎/根辟裂 } \\
\text { Rhizome/Root-splitter }\end{array}$ & CLO-PLA3 & 美洲 America & 有意 Intentionally \\
\hline 1 & 肿柄菊 Tithonia diversifolia & 菊科 Asteraceae & 宿根 Perennial root & Xu \& Qiang, 2011 & 美洲 America & 有意 Intentionally \\
\hline 1 & 落葵薯 Anredera cordifolia & 落葵科 Basellaceae & 根状茎/块茎 Rhizome/Tuber & PIER & 美洲 America & 有意 Intentionally \\
\hline 1 & 马缨丹 Lantana camara & 马鞭草科 Verbenaceae & 根葟 Root turion & CABI & 美洲 America & 有意 Intentionally \\
\hline 1 & 大漂 Pistia stratiotes & 天南星科 Araceae & 匍匐茎 Stolon & Xu \& Qiang, 2011 & 美洲 America & 有意 Intentionally \\
\hline 1 & $\begin{array}{l}\text { 空心莲子草 } \\
\text { Alternanthera philoxeroides }\end{array}$ & 苋科 Amaranthaceae & $\begin{array}{l}\text { 匍匐茎/根状茎/断根 } \\
\text { Stolon/Rhizome/Root fragment }\end{array}$ & Xu \& Qiang, 2011 & 美洲 America & 有意 Intentionally \\
\hline 1 & 五爪金龙 Ipomoea cairica & 旋花科 Convolvulaceae & 匍匐茎/块根 Stolon/Tuberous root & Lin, $2009^{1}$ & 美洲 America & 有意 Intentionally \\
\hline 1 & 凤眼蓝 Eichhornia crassipes & 雨久花科 Pontederiaceae & 匍匐茎 Stolon & Xu \& Qiang, 2011 & 美洲 America & 有意 Intentionally \\
\hline 2 & 红车轴草 Trifolium pratense & 豆科 Leguminosae & 根䢃裂 Root-splitter & CLO-PLA3 & $\begin{array}{l}\text { 非洲/欧洲/亚洲 } \\
\text { Africarica/Europe/Asia }\end{array}$ & 有意 Intentionally \\
\hline 2 & 白车轴草 Trifolium repens & 豆科 Leguminosae & 匍匐茎 Stolon & CLO-PLA3 & $\begin{array}{l}\text { 非洲/欧洲/亚洲 } \\
\text { Africarica/Europe/Asia }\end{array}$ & 有意 Intentionally \\
\hline 2 & 节节麦 Aegilops triuncialis & 禾本科 Gramineae & 分葟 Tiller & Fang F, 2012 & 欧洲 Europe & 有意 Intentionally \\
\hline 2 & 野燕麦 Avena fatua & 禾本科 Gramineae & 分㮐 Tiller & CLO-PLA3 & 欧洲 Europe & 无意 Unintentionally \\
\hline 2 & 巴拉草 Brachiaria mutica & 禾本科 Gramineae & 匍匐茎 Stolon & Xu \& Qiang, 2011 & 非洲 Africarica & 有意 Intentionally \\
\hline 2 & 扁穗雀麦 Bromus catharticus & 禾本科 Gramineae & 分策 Tiller & Cao et al, 2008 & 美洲 America & 有意 Intentionally \\
\hline 2 & 光梗苼藜草 Cenchrus incertus & 禾本科 Gramineae & 分葟 Tiller & Wang, $2009^{2}$ & 美洲 America & 无意 Unintentionally \\
\hline 2 & 红毛草 Melinis repens & 禾本科 Gramineae & 分看 Tiller & CABI & 非洲 Africarica & 有意 Intentionally \\
\hline 2 & 铺地秘 Panicum repens & 禾本科 Gramineae & 分葟 Tiller & Flora of China & 欧洲 Europe & 有意 Intentionally \\
\hline 2 & 两耳草 Paspalum conjugatum & 禾本科 Gramineae & 匍匐茎 Stolon & Flora of China & 美洲 America & 有意 Intentionally \\
\hline
\end{tabular}

1林淳 (2009) 福州市外来入侵植物五爪金龙的危害、生物学特性及风险分析. 硕士学位论文, 福建农林大学, 福州.

${ }^{2}$ 王志新 (2009) 光梗鎂揫草(Cenchrus calyculatus cav.)生物学特性的初步研究, 硕士学位论文, 内蒙古师范大学, 呼和浩特. 
王宁, 李卫芳, 周兵, 间小红. 中国入侵克隆植物入侵性、克隆方式及地理起源. 生物多样性, 2016, 24 (1), 12-19.

http://www.biodiversity-science.net/CN/10.17520/biods.2015190

\begin{tabular}{|c|c|c|c|c|c|c|}
\hline $\begin{array}{l}\text { 入侵等级 } \\
\text { Rank }\end{array}$ & $\begin{array}{l}\text { 种 } \\
\text { Species }\end{array}$ & $\begin{array}{l}\text { 科 } \\
\text { Family }\end{array}$ & 克隆繁殖方式 & 克隆繁殖方式参考资料 & 原产地 & $\begin{array}{l}\text { 引入路径 } \\
\text { Introduction pathway }\end{array}$ \\
\hline 2 & 大米草 Spartina anglica & 禾本科 Gramineae & 根状茎/分葟 Rhizome/Tiller & CLO-PLA3 & 欧洲 Europe & 有意 Intentionally \\
\hline 2 & $\begin{array}{l}\text { 赛葵 } \\
\text { Malvastrum coromandelianum }\end{array}$ & 锦葵科 Malvaceae & 地下芽 Undergound bud & Xu \& Qiang, 2011 & 美洲 America & 无意 Unintentionally \\
\hline 2 & $\begin{array}{l}\text { 南美蟛蜞菊 } \\
\text { Sphagneticola trilobata }\end{array}$ & 菊科 Asteraceae & 匍匐茎 Stolon & PIER & 美洲 America & 有意 Intentionally \\
\hline 2 & 羽芒菊 Tridax procumbens & 菊科 Asteraceae & 地下芽 Undergound bud & Xu \& Qiang, 2011 & 美洲 America & 无意 Unintentionally \\
\hline 2 & 月见草 Oenothera biennis & 柳叶菜科 Onagraceae & $\begin{array}{l}\text { 根蘖/根幦裂 } \\
\text { Root turion/Root-splitter }\end{array}$ & CLO-PLA3 & 美洲 America & 有意 Intentionally \\
\hline 2 & 细叶满江红 Azolla filiculoides & 满江红科 Azollaceae & 断枝 Shoot fragment & CLO-PLA3 & 美洲 America & 有意 Intentionally \\
\hline 2 & 野胡夢卜 Daucus carota & 军形科 Umbelliferae & $\begin{array}{l}\text { 根塾/根幦裂 } \\
\text { Root turion/Root-splitter }\end{array}$ & CLO-PLA3 & 欧洲 Europe & 无意 Unintentionally \\
\hline 2 & 垂序商陆 Phytolacca americana & 商陆科 Phytolaccaceae & 根䢃裂 Root-splitter & CLO-PLA3 & 美洲 America & 有意 Intentionally \\
\hline 2 & 竹节水松 Cabomba caroliniana & 睡莲科 Nymphaeaceae & 断枝 Shoot fragment & Xu \& Qiang, 2011 & 美洲 America & 有意 Intentionally \\
\hline 2 & 仙人掌 Opuntia dillenii & 仙人掌科 Cactaceae & 断枝 Shoot fragment & Xu \& Qiang, 2011 & 美洲 America & 有意 Intentionally \\
\hline 2 & 梨果仙人掌 Opuntia ficus-indica & 仙人掌科 Cactaceae & 断枝 Shoot fragment & Xu \& Qiang, 2011 & 美洲 America & 有意 Intentionally \\
\hline 2 & 单刺仙人掌 Opuntia monacantha & 仙人掌科 Cactaceae & 断枝 Shoot fragment & Xu \& Qiang, 2011 & 美洲 America & 有意 Intentionally \\
\hline 2 & $\begin{array}{l}\text { 刺花莲子草 } \\
\text { Alternanthera pungens }\end{array}$ & 苋科 Amaranthaceae & 匍匐茎 Stolon & PIER & 美洲 America & 无意 Unintentionally \\
\hline 2 & 紫茉莉 Mirabilis jalapa & 紫茉莉科 Nyctaginaceae & 块根 Tuberous root & Flora of China & 美洲 America & 有意 Intentionally \\
\hline 3 & 长叶车前 Plantago lanceolata & 车前科 Plantaginaceae & 分㮐/根䢃裂 Tiller/Root-splitter & CLO-PLA3 & 欧洲 Europe & 有意 Intentionally \\
\hline 3 & 南欧大戟 Euphorbia peplus & 大戟科 Euphorbiaceae & 根葟 Root turion & CLO-PLA3 & 欧洲 Europe & 无意 Unintentionally \\
\hline 3 & 黑荆 Acacia mearnsii & 豆科 Leguminosae & 根菜 Root turion & Fu ZJ, $2005^{1}$ & 大洋洲 Australia & 有意 Intentionally \\
\hline 3 & 山扁豆 Chamaecrista mimosoides & 豆科 Leguminosae & 根菜 Root turion & Xu \& Qiang, 2011 & 美洲 America & 有意 Intentionally \\
\hline 3 & 南美山蚂蝗 Desmodium tortuosum & 豆科 Leguminosae & $\begin{array}{l}\text { 根状茎/断根 } \\
\text { Rhizome/Root fragment }\end{array}$ & Xu \& Qiang, 2011 & 美洲 America & 有意 Intentionally \\
\hline 3 & $\begin{array}{l}\text { 紫花大翼豆 } \\
\text { Macroptilium atropurpureum }\end{array}$ & 豆科 Leguminosae & 不定根 Adventitious root & Flora of China & 美洲 America & 有意 Intentionally \\
\hline 3 & 刺槐 Robinia pseudoacacia & 豆科 Leguminosae & 根藍 Root turion & Xu \& Qiang, 2011 & 美洲 America & 有意 Intentionally \\
\hline 3 & 杂种车轴草 Trifolium hybridum & 豆科 Leguminosae & 根䢃裂 Root-splitter & CLO-PLA3 & 欧洲/亚洲 Europe/Asia & 有意 Intentionally \\
\hline 3 & 珊状臂形草 Brachiaria brizantha & 禾本科 Gramineae & 分藍 Tiller & Deng et al, 2013 & 非洲 Africarica & 有意 Intentionally \\
\hline 3 & 非洲虎尾草 Chloris gayana & 禾本科 Gramineae & $\begin{array}{l}\text { 匍匐茎/不定根 } \\
\text { Stolon/Adventitious root }\end{array}$ & PIER & 非洲 Africarica & 有意 Intentionally \\
\hline 3 & 洋野乘 Panicum dichotomiflorum & 禾本科 Gramineae & 分㰖 Tiller & Flora of China & 美洲 America & 无意 Unintentionally \\
\hline 3 & 大棣 Panicum maximum & 禾本科 Gramineae & 根状茎 Rhizome & Flora of China & 非洲 Africarica & 有意 Intentionally \\
\hline 3 & 毛花雀稗 Paspalum dilatatum & 禾本科 Gramineae & 根状茎 Rhizome & PlantNET & 美洲 America & 有意 Intentionally \\
\hline 3 & $\begin{array}{l}\text { 铺地狼尾草 } \\
\text { Pennisetum clandestinum }\end{array}$ & 禾本科 Gramineae & 匍匐茎/根状茎 Stolon/Rhizome & Flora of China & 非洲 Africarica & 有意 Intentionally \\
\hline 3 & 牧地狼尾草 & 禾本科 Gramineae & 分莧/断茎 Tiller/Stem fragment & CABI & 非洲 Africarica & 有意 Intentionally \\
\hline
\end{tabular}

1付增娟 (2005) 黑荆和银荆的生物入侵研究, 硕士学位论文, 中国林业科学研究院, 北京. 
王宁, 李卫芳, 周兵, 间小红. 中国入侵克隆植物入侵性、克隆方式及地理起源. 生物多样性, 2016, 24 (1), 12-19.

http://www.biodiversity-science.net/CN/10.17520/biods.2015190

\begin{tabular}{|c|c|c|c|c|c|c|}
\hline $\begin{array}{l}\text { 入侵等级 } \\
\text { Rank }\end{array}$ & $\begin{array}{l}\text { 种 } \\
\text { Species }\end{array}$ & $\begin{array}{l}\text { 科 } \\
\text { Family }\end{array}$ & $\begin{array}{l}\text { 克隆繁殖方式 } \\
\text { Clonal reproduction form }\end{array}$ & $\begin{array}{l}\text { 克隆繁殖方式参考资料 } \\
\text { References of clonal reproduction form }\end{array}$ & $\begin{array}{l}\text { 原产地 } \\
\text { Origin }\end{array}$ & $\begin{array}{l}\text { 引入路径 } \\
\text { Introduction pathway }\end{array}$ \\
\hline & Pennisetum polystachion & & & & & \\
\hline 3 & 象草 Pennisetum purpureum & 禾本科 Gramineae & $\begin{array}{l}\text { 分蔝/不定根 } \\
\text { Tiller/Adventitious root }\end{array}$ & Flora of China & 非洲 Africarica & 有意 Intentionally \\
\hline 3 & 黄花㵶 Limnocharis flava & 花萄科 Butomaceae & $\begin{array}{r}\text { 根状茎/花序芽苗 } \\
\text { Rhizome/Plantlet }\end{array}$ & PIER & 美洲 America & 有意 Intentionally \\
\hline 3 & 速生槐叶苹 Salvinia adnata & 槐叶苹科 Salviniaceae & 断枝 Shoot fragment & Xu \& Qiang, 2011 & 美洲 America & 无意 Unintentionally \\
\hline 3 & 洋吊钟 Bryophyllum delagoense & 景天科 Crassulaceae & $\begin{array}{l}\text { 球茎/不定芽 } \\
\text { Corm/Adventitious bud }\end{array}$ & PIER & 非洲 Africarica & 有意 Intentionally \\
\hline 3 & 剑叶金鸡菊 Coreopsis lanceolata & 菊科 Asteraceae & $\begin{array}{l}\text { 分臬/不定根 } \\
\text { Tiller/Adventitious root }\end{array}$ & Zeng et al, 2010 & 美洲 America & 有意 Intentionally \\
\hline 3 & $\begin{array}{l}\text { 海滨月见草 } \\
\text { Oenothera drummondii }\end{array}$ & 柳叶菜科 Onagraceae & 宿根 Perennial root & Xu \& Qiang, 2011 & 美洲 America & 有意 Intentionally \\
\hline 3 & 裂叶月见草 Oenothera laciniata & 柳叶菜科 Onagraceae & 宿根 Perennial root & Xu \& Qiang, 2011 & 美洲 America & 有意 Intentionally \\
\hline 3 & 粉花月见草 Oenothera rosea & 柳叶菜科 Onagraceae & 宿根 Perennial root & Xu \& Qiang, 2011 & 美洲 America & 有意 Intentionally \\
\hline 3 & 火炬树 Rhus typhina & 漆树科 Anacardiaceae & 根菜 Root turion & CLO-PLA3 & 美洲 America & 有意 Intentionally \\
\hline 3 & 颠茄 Atropa belladonna & 茄科 Solanaceae & $\begin{array}{l}\text { 根状茎/根譬裂/根菜 } \\
\text { Rhizome/Root-splitter/Root turion }\end{array}$ & CLO-PLA3 & 欧洲 Europe & 有意 Intentionally \\
\hline 3 & 银毛龙葵 Solanum elaeagnifolium & 茄科 Solanaceae & $\begin{array}{l}\text { 根菜/断根 } \\
\text { Root turion/Root fragment }\end{array}$ & Zhang et al, 2013 & 美洲 America & 无意 Unintentionally \\
\hline 3 & 南美天胡 Hydrocotyle vulgaris & 伞形科 Umbelliferae & $\begin{array}{l}\text { 匍匐茎/分睬/断茎 } \\
\text { Stolon/Tiller/Stem fragment }\end{array}$ & CLO-PLA3 & 美洲 America & 无意 Unintentionally \\
\hline 3 & 球序卷耳 Cerastium glomeratum & 石竹科 Caryophyllaceae & 根䢃裂 Root-splitter & CLO-PLA3 & 欧洲 Europe & 无意 Unintentionally \\
\hline 3 & $\begin{array}{l}\text { 红莲子草 } \\
\text { Alternanthera paronychioides }\end{array}$ & 苋科 Amaranthaceae & 匍匐茎 Stolon & Xu \& Qiang, 2011 & 美洲 America & 有意 Intentionally \\
\hline 3 & 阿拉伯婆婆纳 Veronica persica & 玄参科 Scrophulariaceae & 匍匐茎 Stolon & CLO-PLA3 & 亚洲 Asia & 无意 Unintentionally \\
\hline 3 & 亚麻蒬丝子 Cuscuta epilinum & 旋花科 Convolvulaceae & 断枝 Shoot fragment & AgroAtlas & 欧洲 Europe & 无意 Unintentionally \\
\hline 3 & 月光花 Ipomoea alba & 旋花科 Convolvulaceae & 不定根 Adventitious root & PIER & 美洲 America & 有意 Intentionally \\
\hline 3 & 猫爪藤 Macfadyena unguis-cati & 紫葳科 Bignoniaceae & 块根 Tuberous root & Xu \& Qiang, 2011 & 美洲 America & 有意 Intentionally \\
\hline 4 & 紫苜宿 Medicago sativa & 豆科 Leguminosae & $\begin{array}{l}\text { 根状茎/根擘裂 } \\
\text { Rhizome/Root-splitter }\end{array}$ & CLO-PLA3 & 亚洲 Asia & 有意 Intentionally \\
\hline 4 & 稀脉浮萍 Lemna aequinoctialis & 浮萍科 Lemnaceae & 断枝 Shoot fragment & CABI & 起源不详 Unclear & 有意 Intentionally \\
\hline 4 & 燕麦草 Arrhenatherum elatius & 禾本科 Gramineae & $\begin{array}{l}\text { 根状茎/断茎 } \\
\text { Rhizome/Stem fragment }\end{array}$ & CLO-PLA3 & $\begin{array}{l}\text { 非洲/欧洲/亚洲 } \\
\text { Africarica/Europe/Asia }\end{array}$ & 有意 Intentionally \\
\hline 4 & 野牛草 Buchloe dactyloides & 禾本科 Gramineae & 根状茎/匍匐茎 Rhizome/Stolon & Xu \& Qiang, 2011 & 美洲 America & 有意 Intentionally \\
\hline 4 & 虎尾草 Chloris virgata & 禾本科 Gramineae & 不定根 Adventitious root & PIER & 非洲 Africarica & 有意 Intentionally \\
\hline 4 & 弯叶画眉草 Eragrostis curvula & 禾本科 Gramineae & 分竖 Tiller & Flora of China & 非洲 Africarica & 有意 Intentionally \\
\hline 4 & 芒颖大麦 Hordeum jubatum & 禾本科 Gramineae & 分㹂 Tiller & CLO-PLA3 & $\begin{array}{l}\text { 美洲/欧洲 } \\
\text { America/Europe }\end{array}$ & 有意 Intentionally \\
\hline 4 & 多花黑麦草 Lolium multiflorum & 禾本科 Gramineae & 根状茎/分莧 Rhizome/Tiller & CLO-PLA3 & 欧洲 Europe & 有意 Intentionally \\
\hline 4 & 黑麦草 Lolium perenne & 禾本科 Gramineae & 根状茎/分旙 Rhizome/Tiller & CLO-PLA3 & 欧洲 Europe & 有意 Intentionally \\
\hline 4 & 欧黑麦草 Lolium persicum & 禾本科 Gramineae & 分葟 Tiller & Holman et al, 2006 & 欧洲/亚洲 Europe/Asia & 有意 Intentionally \\
\hline
\end{tabular}


王宁, 李卫芳, 周兵, 间小红. 中国入侵克隆植物入侵性、克隆方式及地理起源. 生物多样性, 2016, 24 (1), 12-19.

http://www.biodiversity-science.net/CN/10.17520/biods.2015190

\begin{tabular}{|c|c|c|c|c|c|c|}
\hline $\begin{array}{l}\text { 入侵等级 } \\
\text { Rank }\end{array}$ & $\begin{array}{l}\text { 种 } \\
\text { Species }\end{array}$ & $\begin{array}{l}\text { 科 } \\
\text { Family }\end{array}$ & $\begin{array}{l}\text { 克隆繁殖方式 } \\
\text { Clonal reproduction form }\end{array}$ & $\begin{array}{l}\text { 克隆繁殖方式参考资料 } \\
\text { References of clonal reproduction form }\end{array}$ & $\begin{array}{l}\text { 原产地 } \\
\text { Origin }\end{array}$ & $\begin{array}{l}\text { 引入路径 } \\
\text { Introduction pathway }\end{array}$ \\
\hline 4 & $\begin{array}{l}\text { 田野黑麦草 } \\
\text { Lolium temulentum var. arvense }\end{array}$ & 禾本科 Gramineae & 分㹂 Tiller & CLO-PLA3 & 欧洲 Europe & 无意 Unintentionally \\
\hline 4 & 梯牧草 Phleum pratense & 禾本科 Gramineae & 分䔣 Tiller & CLO-PLA3 & 欧洲 Europe & 有意 Intentionally \\
\hline 4 & 加拿大早熟禾 Poa compressa & 禾本科 Gramineae & 根状茎/分䅼 Rhizome/Tiller & CLO-PLA3 & 欧洲 Europe & 有意 Intentionally \\
\hline 4 & 黑麦 Secale cereale & 禾本科 Gramineae & 分藍 Tiller & CLO-PLA3 & 亚洲 Asia & 有意 Intentionally \\
\hline 4 & 棕叶狗尾草 Setaria palmifolia & 禾本科 Gramineae & 根状茎 Rhizome & Flora of China & 非洲 Africarica & 有意 Intentionally \\
\hline 4 & 幽狗尾草 Setaria parviflora & 禾本科 Gramineae & 根状茎 Rhizome & Flora of China & 美洲 America & 无意 Unintentionally \\
\hline 4 & 草胡椒 Peperomia pellucida & 胡椒科 Piperaceae & 不定根 Adventitious root & Xu \& Qiang, 2011 & 美洲 America & 无意 Unintentionally \\
\hline 4 & 菊苣 Cichorium intybus & 菊科 Asteraceae & $\begin{array}{l}\text { 根塾/根擘裂 } \\
\text { Root turion/Root-splitter }\end{array}$ & CLO-PLA3 & 欧洲 Europe & 有意 Intentionally \\
\hline 4 & 菊芋 Helianthus tuberosus & 菊科 Asteraceae & 根状茎/块茎 Rhizome/Tuber & CLO-PLA3 & 美洲 America & 有意 Intentionally \\
\hline 4 & 裸柱菊 Soliva anthemifolia & 菊科 Asteraceae & 根状茎/匍匐茎 Rhizome/Stolon & Xu et al, 2011 & 美洲 America & 无意 Unintentionally \\
\hline 4 & 药用蒲公英 Taraxacum officinale & 菊科 Asteraceae & $\begin{array}{l}\text { 根䔗/根䢃裂 } \\
\text { Root turion/Root-splitter }\end{array}$ & CLO-PLA3 & 欧洲 Europe & 无意 Unintentionally \\
\hline 4 & 小酸模 Rumex acetosella & 蓼科 Polygonaceae & $\begin{array}{l}\text { 根状茎/根菜/根幦裂 } \\
\text { Rhizome/Root turion/Root-splitter }\end{array}$ & CLO-PLA3 & 欧洲/亚洲 Europe/Asia & 有意 Intentionally \\
\hline 4 & 灯笼果 Physalis peruviana & 茄科 Solanaceae & 根状茎 Rhizome & FRPS & 美洲 America & 有意 Intentionally \\
\hline 4 & 香附子 Cyperus rotundus & 莎草科 Cyperaceae & 块茎 Tuber & CLO-PLA3 & $\begin{array}{l}\text { 非洲/欧洲/亚洲 } \\
\text { Africarica/Europe/Asia }\end{array}$ & 有意 Intentionally \\
\hline 4 & 绿独行菜 Lepidium campestre & 十字花科 Cruciferae & 根䢃裂 Root-splitter & CLO-PLA3 & 欧洲/亚洲 Europe/Asia & 无意 Unintentionally \\
\hline 4 & 豆瓣菜 Nasturtium officinale & 十字花科 Cruciferae & $\begin{array}{l}\text { 匍匐茎/断枝 } \\
\text { Stolon/Shoot fragment }\end{array}$ & CLO-PLA3 & 欧洲/亚洲 Europe/Asia & 有意 Intentionally \\
\hline 4 & 葱莲 Zephyranthes candida & 石蒜科 Amaryllidaceae & 鳞茎 Bulb & PBS & 美洲 America & 有意 Intentionally \\
\hline 4 & 非莲 Zephyranthes carinata & 石赫科 Amaryllidaceae & 鳞茎 Bulb & PBS & 美洲 America & 有意 Intentionally \\
\hline 4 & 鹅肠菜 Myosoton aquaticum & 石竹科 Caryophyllaceae & 根状茎/匍匐茎 Rhizome/Stolon & CLO-PLA3 & 欧洲 Europe & 无意 Unintentionally \\
\hline 4 & 直立婆婆纳 Veronica arvensis & 玄参科 Scrophulariaceae & 匍匐茎 Stolon & Wu HL, 2006 & 欧洲/亚洲 Europe/Asia & 无意 Unintentionally \\
\hline 4 & 婆婆纳 Veronica polita & 玄参科 Scrophulariaceae & 匍匐茎 Stolon & Wu HL, 2006 & 亚洲 Asia & 无意 Unintentionally \\
\hline 4 & 红花酢浆草 Oxalis corymbosa & 酢浆草科 Oxalidaceae & 鳞茎 Bulb & Xu \& Qiang, 2011 & 美洲 America & 有意 Intentionally \\
\hline 4 & 宽叶酢浆草 Oxalis latifolia & 酢浆草科 Oxalidaceae & 鳞茎 Bulb & CLO-PLA3 & 美洲 America & 有意 Intentionally \\
\hline 5 & 假非 Nothoscordum gracile & 百合科 Liliaceae & 鳞茎 Bulb & PBS & 美洲 America & 有意 Intentionally \\
\hline 5 & 凤尾兰 Yucca gloriosa & 百合科 Liliaceae & 根蕃Root turion & Flora of North America & 美洲 America & 有意 Intentionally \\
\hline 5 & 留兰香 Mentha spicata & 唇形科 Lamiaceae & 根状茎 Rhizome & CLO-PLA3 & $\begin{array}{l}\text { 非洲/欧洲/亚洲 } \\
\text { Africarica/Europe/Asia }\end{array}$ & 有意 Intentionally \\
\hline 5 & 匍根大戟 Euphorbia serpens & 大戟科 Euphorbiaceae & 匍匐茎 Stolon & Flora of China & 美洲 America & 无意 Unintentionally \\
\hline 5 & 银荆 Acacia dealbata & 豆科 Leguminosae & 根菜 Root turion & Fu ZJ, $2005^{1}$ & 大洋洲 Australia & 有意 Intentionally \\
\hline 5 & 紫穗槐 Amorpha fruticosa & 豆科 Leguminosae & 根菜 Root turion & Illinois Wildflowers & 美洲 America & 有意 Intentionally \\
\hline 5 & 绣球小冠花 Coronilla varia & 豆科 Leguminosae & 根状茎/根葟/根䢃裂 & CLO-PLA3 & 欧洲 Europe & 有意 Intentionally \\
\hline
\end{tabular}

1付增娟 (2005) 黑荆和银荆的生物入侵研究. 硕士学位论文, 中国林业科学研究院, 北京. 
王宁, 李卫芳, 周兵, 间小红. 中国入侵克隆植物入侵性、克隆方式及地理起源. 生物多样性, 2016, 24 (1), 12-19.

http://www.biodiversity-science.net/CN/10.17520/biods.2015190

\begin{tabular}{|c|c|c|c|c|c|c|}
\hline $\begin{array}{l}\text { 入侵等级 } \\
\text { Rank }\end{array}$ & $\begin{array}{l}\text { 种 } \\
\text { Species }\end{array}$ & $\begin{array}{l}\text { 科 } \\
\text { Family }\end{array}$ & $\begin{array}{l}\text { 克隆繁殖方式 } \\
\text { Clonal reproduction form }\end{array}$ & $\begin{array}{l}\text { 克隆繁殖方式参考资料 } \\
\text { References of clonal reproduction form }\end{array}$ & $\begin{array}{l}\text { 原产地 } \\
\text { Origin }\end{array}$ & $\begin{array}{l}\text { 引入路径 } \\
\text { Introduction pathway }\end{array}$ \\
\hline & & & Rhizome/Root turion/Root-splitte & & & \\
\hline 5 & 大翼豆 Macroptilium lathyroides & 豆科 Leguminosae & 不定根 Adventitious root & Teng \& Lai, 2013 & 美洲 America & 有意 Intentionally \\
\hline 5 & 假含羞草 Neptunia plena & 豆科 Leguminosae & 不定根 Adventitious root & PIER & 美洲 America & 有意 Intentionally \\
\hline 5 & 草莓车轴草 Trifolium fragiferum & 豆科 Leguminosae & 匍匐茎 Stolon & CLO-PLA3 & $\begin{array}{l}\text { 非洲/欧洲/亚洲 } \\
\text { Africarica/Europe/Asia }\end{array}$ & 有意 Intentionally \\
\hline 5 & 地毯草 Axonopus compressue & 禾本科 Gramineae & 匍匐茎 Stolon & PIER & 美洲 America & 有意 Intentionally \\
\hline 5 & 香根草 Chrysopogon zizanioides & 禾本科 Gramineae & 分葟 Tiller & Xu \& Qiang, 2011 & 亚洲 Asia & 有意 Intentionally \\
\hline 5 & 香茅 Cymbopogon citratus & 禾本科 Gramineae & 分㹂 Tiller & Flora of China & 亚洲 Asia & 有意 Intentionally \\
\hline 5 & 亚香茅 Cymbopogon nardus & 禾本科 Gramineae & 分覧 Tiller & CABI & 亚洲 Asia & 有意 Intentionally \\
\hline 5 & 弯穗草 Dinebra retroflexa & 禾本科 Gramineae & 分蓧 Tiller & Flora of China & 非洲/亚洲 Africa/Asia & 有意 Intentionally \\
\hline 5 & 皱稃草 Ehrharta erecta & 禾本科 Gramineae & 不定根 Adventitious root & CABI & 非洲 Africa & 有意 Intentionally \\
\hline 5 & 苇状羊茅 Festuca arundinacea & 禾本科 Gramineae & 根状茎/分榄 Rhizome/Tiller & CLO-PLA3 & 欧洲 Europe & 有意 Intentionally \\
\hline 5 & 球茎大麦 Hordeum bulbosum & 禾本科 Gramineae & 根状茎/分臬 Rhizome/Tiller & CLO-PLA3 & 欧洲 Europe & 有意 Intentionally \\
\hline 5 & 疏花黑麦草 Lolium remotum & 禾本科 Gramineae & 分藍 Tiller & CLO-PLA3 & 欧洲 Europe & 有意 Intentionally \\
\hline 5 & 假牛鞭草 Parapholis incurva & 禾本科 Gramineae & 匍匐茎 Stolon & Ding et al, 2013 & 欧洲 Europe & 无意 Unintentionally \\
\hline 5 & 裂颖雀稗 Paspalum fimbriatum & 禾本科 Gramineae & 分蕃 Tiller & Flora of China & 美洲 America & 有意 Intentionally \\
\hline 5 & $\begin{array}{l}\text { 棱稃雀稗 } \\
\text { Paspalum malacophyllum }\end{array}$ & 禾本科 Gramineae & 根状茎 Rhizome & Flora of China & 美洲 America & 有意 Intentionally \\
\hline 5 & 百喜草 Paspalum notatum & 禾本科 Gramineae & 根状茎/匍匐茎 Rhizome/Stolon & USDA & 美洲 America & 有意 Intentionally \\
\hline 5 & 开穗雀稗 Paspalum paniculatum & 禾本科 Gramineae & 根状茎 Rhizome & PlantNET & 美洲 America & 有意 Intentionally \\
\hline 5 & 皱稃雀稗 Paspalum plicatulum & 禾本科 Gramineae & 根状茎 Rhizome & USDA & 美洲 America & 有意 Intentionally \\
\hline 5 & 丝毛雀稗 Paspalum urvillei & 禾本科 Gramineae & 根状茎 Rhizome & CABI & 美洲 America & 有意 Intentionally \\
\hline 5 & 粗秆雀稗 Paspalum virgatum & 禾本科 Gramineae & 根状茎 Rhizome & FRPS & 美洲 America & 有意 Intentionally \\
\hline 5 & 细曧草 Phalaris minor & 禾本科 Gramineae & 分覧 Tiller & Guo et al, 2010 & $\begin{array}{l}\text { 非洲/欧洲/亚洲 } \\
\text { Africarica/Europe/Asia }\end{array}$ & 有意 Intentionally \\
\hline 5 & 奇虉草 Phalaris paradoxa & 禾本科 Gramineae & 分蒕 Tiller & Guo et al, 2010 & $\begin{array}{l}\text { 非洲/欧洲/亚洲 } \\
\text { Africarica/Europe/Asia }\end{array}$ & 有意 Intentionally \\
\hline 5 & 南非鸽草 Setaria sphacelata & 禾本科 Gramineae & 根状茎 Rhizome & PIER & 非洲 Africa & 有意 Intentionally \\
\hline 5 & 黑高粱 Sorghum × almum & 禾本科 Gramineae & 根状茎 Rhizome & FRPS & 美洲 America & 无意 Unintentionally \\
\hline 5 & 苏丹草 Sorghum sudanense & 禾本科 Gramineae & 分菳 Tiller & Ding \& Shen, 2001 & 非洲 Africa & 有意 Intentionally \\
\hline 5 & $\begin{array}{l}\text { 具枕鼠尾粟 } \\
\text { Sporobolus pyramidatus }\end{array}$ & 禾本科 Gramineae & 分蒕 Tiller & PIER & 美洲 America & 有意 Intentionally \\
\hline 5 & 药葵 Althaea officinalis & 锦葵科 Malvaceae & 根状茎 Rhizome & CLO-PLA3 & 欧洲/亚洲 Europe/Asia & 有意 Intentionally \\
\hline 5 & $\begin{array}{l}\text { 大叶落地生根 } \\
\text { Bryophyllum daigremontianum }\end{array}$ & 景天科 Crassulaceae & 不定芽 Adventitious bud & PIER & 非洲 Africa & 有意 Intentionally \\
\hline 5 & 落地生根 Bryophyllum pinnatum & 景天科 Crassulaceae & 不定芽 Adventitious bud & PIER & 非洲 Africa & 有意 Intentionally \\
\hline 5 & 春黄菊 Anthemis tinctoria & 菊科 Asteraceae & 根幦裂 Root-splitter & CLO-PLA3 & 欧洲 Europe & 有意 Intentionally \\
\hline 5 & 金腰箭舅 Calyptocarpus vialis & 菊科 Asteraceae & 不定根 Adventitious root & PIER & 美洲 America & 无意 Unintentionally \\
\hline
\end{tabular}


王宁, 李卫芳, 周兵, 间小红. 中国入侵克隆植物入侵性、克隆方式及地理起源. 生物多样性, 2016, 24 (1), 12-19.

http://www.biodiversity-science.net/CN/10.17520/biods.2015190

\begin{tabular}{|c|c|c|c|c|c|c|}
\hline $\begin{array}{l}\text { 入侵等级 } \\
\text { Rank }\end{array}$ & $\begin{array}{l}\text { 种 } \\
\text { Species }\end{array}$ & $\begin{array}{l}\text { 科 } \\
\text { Family }\end{array}$ & $\begin{array}{l}\text { 克隆繁殖方式 } \\
\text { Clonal reproduction form }\end{array}$ & $\begin{array}{l}\text { 克隆繁殖方式参考资料 } \\
\text { References of clonal reproduction form }\end{array}$ & $\begin{array}{l}\text { 原产地 } \\
\text { Origin }\end{array}$ & $\begin{array}{l}\text { 引入路径 } \\
\text { Introduction pathway }\end{array}$ \\
\hline 5 & 金鸡菊 Coreopsis basalis & 菊科 Asteraceae & 宿根 Perennial root & Guo YX, $2012^{1}$ & 美洲 America & 有意 Intentionally \\
\hline 5 & 大花金鸡菊 Coreopsis grandiflora & 菊科 Asteraceae & 宿根 Perennial root & Guo YX, $2012^{1}$ & 美洲 America & 有意 Intentionally \\
\hline 5 & $\begin{array}{l}\text { 蓝花野泀蒿 } \\
\text { Crassocephalum rubens }\end{array}$ & 菊科 Asteraceae & 不定根 Adventitious root & Xu \& Qiang, 2011 & 非洲 Africa & 有意 Intentionally \\
\hline 5 & $\begin{array}{l}\text { 白花地胆草 } \\
\text { Elephantopus tomentosus }\end{array}$ & 菊科 Asteraceae & 根状茎 Rhizome & Flora of China & 美洲 America & 无意 Unintentionally \\
\hline 5 & $\begin{array}{l}\text { 大麻叶泽兰 } \\
\text { Eupatorium cannabinum }\end{array}$ & 菊科 Asteraceae & 根状茎 Rhizome & CLO-PLA3 & 欧洲 Europe & 有意 Intentionally \\
\hline 5 & $\begin{array}{l}\text { 裸冠菊 } \\
\text { Gymnocoronis spilanthoides }\end{array}$ & 菊科 Asteraceae & 断茎 Stem fragment & PIER & 美洲 America & 有意 Intentionally \\
\hline 5 & 滨菊 Leucanthemum vulgare & 菊科 Asteraceae & 分蓞 Tiller & CLO-PLA3 & 欧洲 Europe & 有意 Intentionally \\
\hline 5 & $\begin{array}{l}\text { 假地胆草 } \\
\text { Pseudephantopus spicatus }\end{array}$ & 菊科 Asteraceae & 地下芽 Undergound bud & Xu \& Qiang, 2011 & 美洲 America & 无意 Unintentionally \\
\hline 5 & 金光菊 Rudbeckia laciniata & 菊科 Asteraceae & 根状茎/分葟 Rhizome/Tiller & CLO-PLA3 & 美洲 America & 有意 Intentionally \\
\hline 5 & 串叶松香草 Silphium perfoliatum & 菊科 Asteraceae & 根状茎 Rhizome & CLO-PLA3 & 美洲 America & 有意 Intentionally \\
\hline 5 & 翅果裸柱菊 Soliva pterosperma & 菊科 Asteraceae & 匍匐茎 Stolon & PIER & 美洲 America & 无意 Unintentionally \\
\hline 5 & $\begin{array}{l}\text { 伞房匹菊 } \\
\text { Tanacetum parthenifolium }\end{array}$ & 菊科 Asteraceae & 分蒕 Tiller & CLO-PLA3 & 亚洲 Asia & 有意 Intentionally \\
\hline 5 & 芦莉草 Ruellia tuberosa & 爵床科 Acanthaceae & 块根 Tuberous root & Flora of China & 美洲 America & 有意 Intentionally \\
\hline 5 & 珊瑚藤 Antigonon leptopus & 蓼科 Polygonaceae & 块根 Tuberous root & PIER & 美洲 America & 有意 Intentionally \\
\hline 5 & 黄花月见草 Oenothera glazioviana & 柳叶菜科 Onagraceae & 宿根 Perennial root & Xu \& Qiang, 2011 & 欧洲 Europe & 有意 Intentionally \\
\hline 5 & 长毛月见草 Oenothera villosa & 柳叶菜科 Onagraceae & 宿根 Perennial root & Xu \& Qiang, 2011 & 美洲 America & 有意 Intentionally \\
\hline 5 & 短序落葵薯 Anredera scandens & 落葵科 Basellaceae & 块根 Tuberous root & Flora of China & 美洲 America & 有意 Intentionally \\
\hline 5 & 蔓马缨丹 Lantana montevidensis & 马鞭草科 Verbenaceae & 不定根 Adventitious root & PIER & 美洲 America & 有意 Intentionally \\
\hline 5 & 黄木犀草 Reseda lutea & 木犀草科 Resedaceae & $\begin{array}{l}\text { 根鼡/根䢃裂 } \\
\text { Root turion/Root-splitter }\end{array}$ & CLO-PLA3 & $\begin{array}{l}\text { 非洲/欧洲/亚洲 } \\
\text { Africarica/Europe/Asia }\end{array}$ & 有意 Intentionally \\
\hline 5 & $\begin{array}{l}\text { 五叶地锦 } \\
\text { Parthenocissus quinquefolia }\end{array}$ & 葡萄科 Vitaceae & 断茎 Stem fragment & Liu et al, 2008 & 美洲 America & 有意 Intentionally \\
\hline 5 & 轮叶节节菜 Rotala mexicana & 千屈菜科 Lythraceae & 匍匐茎 Stolon & Kerala Plants & 美洲 America & 有意 Intentionally \\
\hline 5 & 北美刺龙葵 Solanum carolinense & 茄科 Solanaceae & $\begin{array}{l}\text { 根状茎/断根 } \\
\text { Rhizome/Root fragment }\end{array}$ & Penn State Extension & 美洲 America & 无意 Unintentionally \\
\hline 5 & 黄香附 Cyperus esculentus & 莎草科 Cyperaceae & 根状茎/块茎 Rhizome/Tuber & CLO-PLA3 & $\begin{array}{l}\text { 非洲/欧洲/亚洲 } \\
\text { Africarica/Europe/Asia }\end{array}$ & 有意 Intentionally \\
\hline 5 & 风车草 Cyperus involucratus & 莎草科 Cyperaceae & 根状茎 Rhizome & Flora of China & 非洲 Africarica & 有意 Intentionally \\
\hline 5 & 辣根 Armoracia rusticana & 十字花科 Cruciferae & 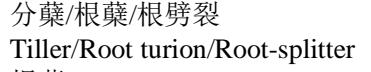 & CLO-PLA3 & 欧洲 Europe & 有意 Intentionally \\
\hline 5 & 二行芥 Diplotaxis muralis & 十字花科 Cruciferae & 根葟 Root turion & CLO-PLA3 & 欧洲 Europe & 无意 Unintentionally \\
\hline 5 & 龙舌兰 Agave americana & 石蒜科 Amaryllidaceae & 根状茎/珠芽 Rhizome/Bulbil & CLO-PLA3 & 美洲 America & 有意 Intentionally \\
\hline 5 & 剑麻 Agave sisalana & 石蒜科 Amaryllidaceae & 根状茎/珠芽 Rhizome/Bulbil & PIER & 美洲 America & 有意 Intentionally \\
\hline
\end{tabular}

1 郭运雪 (2012) 3 种金鸡菊的光合生理特性及抗早性研究. 硕士学位论文, 浙江农林大学, 临安. 
王宁, 李卫芳, 周兵, 闻小红. 中国入侵克隆植物入侵性、克隆方式及地理起源. 生物多样性, 2016, 24 (1), 12-19.

http://www.biodiversity-science.net/CN/10.17520/biods.2015190

\begin{tabular}{|c|c|c|c|c|c|c|}
\hline $\begin{array}{l}\text { 入侵等级 } \\
\text { Rank }\end{array}$ & $\begin{array}{l}\text { 种 } \\
\text { Species }\end{array}$ & $\begin{array}{l}\text { 科 } \\
\text { Family }\end{array}$ & 克隆繁殖方式 & 克隆繁殖方式参考资料 & $\begin{array}{l}\text { 原产地 } \\
\text { Origin }\end{array}$ & 引入路径 \\
\hline$\overline{5}$ & 花朱顶红 Hippeastrum vittatum & 石蒜科 Amaryllidaceae & 鳞茎 Bulb & FRPS & 美洲 America & 有意 Intentionally \\
\hline 5 & 肥㿝草 Saponaria officinalis & 石竹科 Caryophyllaceae & $\begin{array}{l}\text { 根状茎/根箵 } \\
\text { Rhizome/Root turion }\end{array}$ & CLO-PLA3 & 欧洲/亚洲 Europe/Asia & 有意 Intentionally \\
\hline 5 & 水蕴草 Egeria densa & 水鳖科 Hydrocharitaceae & $\begin{array}{l}\text { 断枝/磷芽 } \\
\text { Shoot fragment/Turion }\end{array}$ & CLO-PLA3 & 美洲 America & 无意 Unintentionally \\
\hline 5 & 千年芋 Xanthosoma sagittifolium & 天南星科 Araceae & 块茎 Tuber & PIER & 美洲 America & 有意 Intentionally \\
\hline 5 & 量天尺 Hylocereus undatus & 仙人掌科 Cactaceae & 断茎 Stem fragment & PIER & 美洲 America & 有意 Intentionally \\
\hline 5 & 木麒麟 Pereskia aculeata & 仙人掌科 Cactaceae & 断茎 Stem fragment & PIER & 美洲 America & 有意 Intentionally \\
\hline 5 & $\begin{array}{l}\text { 粉绿狐尾藻 } \\
\text { Myriophyllum aquaticum }\end{array}$ & $\begin{array}{l}\text { 小二仙草科 } \\
\text { Haloragaceae }\end{array}$ & 断枝 Shoot fragment & WSDE & 美洲 America & 有意 Intentionally \\
\hline 5 & 暻柳穿鱼 Cymbalaria muralis & 玄参科 Scrophulariaceae & 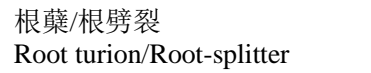 & CLO-PLA3 & 欧洲 Europe & 有意 Intentionally \\
\hline 5 & 毛地黄 Digitalis purpurea & 玄参科 Scrophulariaceae & 分㮐 Tiller & CLO-PLA3 & 欧洲 Europe & 有意 Intentionally \\
\hline 5 & 杯花菟丝子 Cuscuta approximata & 旋花科 Convolvulaceae & 断茎 Stem fragment & Dilber, $2008^{1}$ & $\begin{array}{l}\text { 非洲/欧洲/亚洲 } \\
\text { Africarica/Europe/Asia }\end{array}$ & 无意 Unintentionally \\
\hline 5 & 原野菟丝子 Cuscuta campestris & 旋花科 Convolvulaceae & 断茎 Stem fragment & PIER & 美洲 America & 无意 Unintentionally \\
\hline 5 & 变色牵牛 Ipomoea indica & 旋花科 Convolvulaceae & 断茎 Stem fragment & PIER & 美洲 America & 有意 Intentionally \\
\hline 5 & 七爪龙 Ipomoea mauritiana & 旋花科 Convolvulaceae & 块根 Tuberous root & Flora of China & 起源不详 Unclear & 有意 Intentionally \\
\hline 5 & 木玫瑰 Merremia tuberosa & 旋花科 Convolvulaceae & 断根 Root fragment & KALA & 美洲 America & 有意 Intentionally \\
\hline 5 & 洋竹草 Callisia repens & $\begin{array}{l}\text { 鸭 跖 草 } \\
\text { Commelinaceae }\end{array}$ & 匍匐茎 Stolon & Flora of China & 美洲 America & 有意 Intentionally \\
\hline 5 & 紫竹梅 Tradescantia pallida & $\begin{array}{l}\text { 鸭 跖 草 } \\
\text { Commelinaceae }\end{array}$ & 匍匐茎 Stolon & CABI & 美洲 America & 有意 Intentionally \\
\hline 5 & 吊竹梅 Tradescantia zebrina & $\begin{array}{l}\text { 鸭 跖 草 } \\
\text { Commelinaceae }\end{array}$ & 匍匐茎 Stolon & Flora of China & 美洲 America & 有意 Intentionally \\
\hline 5 & $\begin{array}{l}\text { 雄黄兰 } \\
\text { Crocosmia } \times \text { crocosmiiflora }\end{array}$ & 鸢尾科 Iridaceae & 球茎 Corm & PBS & 非洲 Africarica & 有意 Intentionally \\
\hline 5 & 黄菖蒲 Iris pseudacorus & 窎尾科 Iridaceae & 分菜 Tiller & CLO-PLA3 & 欧洲 Europe & 有意 Intentionally \\
\hline 5 & 再力花 Thalia dealbata & 竹芋科 Marantaceae & 块茎 Tuber & USDA & 美洲 America & 有意 Intentionally \\
\hline 5 & 聚合草 Symphytum officinale & 紫草科 Boraginaceae & 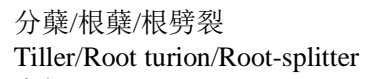 & CLO-PLA3 & 欧洲 Europe & 有意 Intentionally \\
\hline 5 & $\begin{array}{l}\text { 夜香紫茉莉 } \\
\text { Oxybaphus nyctagineus }\end{array}$ & 紫茉莉科 Nyctaginaceae & 宿根 Perennial root & Ohio Perennial \& Biennial Weed Guide & 美洲 America & 有意 Intentionally \\
\hline 5 & 大花酢浆草 Oxalis bowiei & 酢浆草科 Oxalidaceae & 鳞茎 Bulb & FRPS & 非洲 Africarica & 有意 Intentionally \\
\hline 5 & 紫叶酢浆草 Oxalis triangularis & 酢浆草科 Oxalidaceae & 鳞茎 Bulb & PBS & 美洲 America & 有意 Intentionally \\
\hline
\end{tabular}

AgroAtlas, Interactive Agricultural Ecological Atlas of Russia and Neighboring Countries; CABI, Centre for Agriculture and Biosciences International; CLO-PLA3, CLOnal PLAnts, version 3; FRPS, Flora USDA, United States Department of Agriculture; WSDE, Washington State Department of Ecology. 


\section{参考文献}

Cao QG, Zhang YJ, Qu LS (2008) Reproduction strategy of flat bromegrass in Yunnan Province. Seed, 27, 101-103. (in Chinese) [曹清国, 张幽静, 区力松 (2008) 云南地区扁穗雀麦繁殖对 策研究. 种子, 27, 101-103.]

Centre for Agriculture and Biosciences International (CABI) (2015) Invasive Species Compendium. http://www.cabi.org/isc (accessed 2015-06-20)

Chinese Academy of Sciences (2004) Flora Reipublicae Popularis Sinicae (FRPS). [中国科学院 (2004) 《中国植物志》全文电子版网站.] http://frps.eflora.cn (accessed 2015-06-22)

Deng NJ, Dong ZS, Li DR, Pu GB, Liu JP, Liu JX, Li M, Guan HW, Zhang JH (2013) A comparative experiment of 4 introduced species of Brachiaria genus in Xishuangbanna. Pratacultural Science, 30, 821-825. (in Chinese with English abstract) [邓妮娟, 董仲生, 李德 荣, 普光保, 刘建平, 刘菊香, 李明, 管红伟, 张建虎 (2013) 西双版纳州4种臂形草属牧草引 种试验. 草业科学, 30, 821-825.]

Ding CL, Shen YX (2001) The growth expression of ten Sudan grass cultivars in southern region of China. Grassland of China, 23, 72-78. (in Chinese with English abstract) [丁成龙, 沈益新 (2001) 10 个苏丹草品种在南方的生长表现. 中国草地, 23, 72-78.]

Ding XJ, Han H, Pang CJ, Ma YX, Cui DL, Fan CC (2013) Spatial distribution pattern with fractal theory for Carex kobomugi and Parapholis incurve populations. Journal of Zhejiang A \& F University, 30, 220-225. (in Chinese with English abstract) [丁雪娇, 韩红, 庞彩菊, 马玉 心, 崔大练, 范彩彩 (2013) 砂砧薹草和假牛鞭草种群空间分布格局的分形特征. 浙江农林 大学学报, 30, 220-225.]

Fang F (2012) Ecological Adaptability of Tausch’s Goatgrass (Aegilops tauschii Coss.). PhD dissertation, Chinese Academy of Agricultural Sciences, Beijing. (in Chinese with English abstract) [房锋 (2002) 节节麦(Aegilops tauschii Coss.)生态适应性. 博士学位论文, 中国农 业科学院, 北京.]

Guo YQ, Zhao GJ, Chen Y, Yue Y, Wang LX (2010) Infestation of invasive weed in crop field in Yunan. Southwest China Journal of Agricultural Sciences, 23, 1352-1355. (in Chinese with English abstract) [郭怡卿, 赵国晶, 陈勇, 岳英, 王玲仙 (2010) 云南农田外来杂草及其危害 现状. 西南农业学报, 23, 1352-1355.]

Hilty J (2015) Illinois Wildflowers. http://www.illinoiswildflowers.info (accessed 2015-06-22)

Holman JD, Bussan AJ, Maxwell BD, Miller PR, Mickelson JA (2006) Persian darnel (Lolium persicum) fecundity response to spring wheat, canola, and sunflower interference. Weed Technology, 20, 430-437.

Institute of Pacific Islands Forestry (2013) Pacific Island Ecosystems at Risk (PIER). http://www.hear.org/pier (accessed 2015-06-20)

Interactive Agricultural Ecological Atlas of Russia and Neighboring Countries (AgroAtlas) (2009) Economic Plants and their Diseases, Pests and Weeds. http://www.agroatlas.ru/en/content/weeds/\#C (accessed 2015-06-22)

Kalaupapa National Historical Park (KALA) (2012) Invasive Plant Field Guide. https://science.na ture.nps.gov/im/units/pacn/assets/docs/Invasive_Species_Cards_and_Calendars_PBIN/NPS_C ARDS_KALA_12112012_final.pdf (accessed 2015-06-20)

Kerala Forest Research Institute (2011) Kerala Plants. http://keralaplants.in/index.html (accessed
2015-06-20)

Klimešová J, Klimeš L (2013) Clo-Pla3-database of clonal growth of plants from Central Europe. http://clopla.butbn.cas.cz (accessed 2015-06-20)

Liu MJ, Xu GF, Wang HS, Zhu XL (2008) The invasive characteristic of Parthenocissus quinquefolia (L.) Planch. Acta Agriculturae Boreali-occidentalis Sinica, 17, 234-237. (in Chinese with English abstract) [刘明久, 许桂芳, 王鸿升, 朱晓利 (2008) 美国地锦入侵特性 研究. 西北农业学报, 17, 234-237.]

Lü LY, Zhao Y, Wang HX, Wang W (2011) Effects of mowing on plant regrowth and reproduction characteristics of invasive Cenchrus pauciflorus. Pratacultural Science, 28, 100104. (in Chinese with English abstract) [吕林有, 赵艳, 王海新, 王巍 (2011) 刈割对入侵植物 少花薮藜草再生生长及繁殖特性的影响. 草业科学, 28, 100-104.]

Missouri Botanical Garden (2008) eFloras. http://www.efloras.org [accessed 2015-06-22]

Quan GM, Mao DJ, Zhang JE, Xie JF, Xu HQ (2011) Reproductive capacity and seed germination characteristics of Chromolaena odorata. Ecology and Environmental Sciences, 20, 72-78. (in Chinese with English abstract) [全国明, 毛丹鹃, 章家恩, 谢俊芳, 徐华勤 (2011) 飞机草的繁殖能力与种子的萌发特性. 生态环境学报, 20, 72-78.]

Royal Botanic Gardens and Domain Trust, Sydney (2004) The NSW Plant Information Network System (PlantNET). http://plantnet.rbgsyd.nsw.gov.au (accessed 2015-06-20)

Teng SH, Lai ZQ (2013) High yield cultivation and untilization of excellent Legume forage Macroptilium lathyroides. Shanghai Journal of Animal Husbandry and Veterinary Medicine, 60(5), 52-53. (in Chinese) [滕少花, 赖志强 (2013) 优良豆科牧草大翼豆高产栽培与利用. 上海畜牧兽医通讯, 60, 52-53.]

The Ohio State University (2006) Ohio Perennial \& Biennial Weed Guide. http://www.oardc.ohio-state.edu/weedguide/listall.asp (accessed 2015-06-20)

The Pacific Bulb Society (PBS) (2002) Photographs and Information. http://www.pacificbulbsociety.org (accessed 2015-06-20)

The Pennsylvania State University (2015) Penn State Extension. http://extension.psu.edu (accessed 2015-06-20)

Tian XS, Yue MF, Feng L, Yang CH, Yang HM (2010) Characteristics of alien weed Bidens alba. Jiangsu Agricultural Sciences, 38, 174-175. (in Chinese) [田兴山, 岳茂峰, 冯莉, 杨彩 宏, 杨红梅 (2010) 外来入侵杂草白花鬼针草的特征特性. 江苏农业科学, 38, 174-175.]

United States Department of Agriculture (USDA) (2015) Fact Sheets \& Plant Guides. http://plants.usda.gov/java/factSheet (accessed 2015-06-22)

Washington State Department of Ecology (WSDE) (2015) Non-native, Invasive, Freshwater Plants. http://www.ecy.wa.gov/Programs/wq/plants/weeds/index.html (accessed 2015-06-22)

Wu HR (2006) Quantitative Survey on Exotic Weeds in Nanjing and Comparison of Invasive Characteristics of Exotic Weeds of Veronica. PhD dissertation, Nanjing Agricultural University, Nanjing. (in Chinese with English abstract) [吴海荣 (2006) 南京地区外来杂草调 查及婆波纳属外来杂草入侵性特征比较研究. 博士学位论文, 南京农业大学, 南京.]

Xu HG, Qiang S (2011) Chinese Invasive Alien Species. Science Press, Beijing. (in Chiness) [徐 海根, 强胜 (2011) 中国外来入侵生物. 科学出版社, 北京.] 
王宁, 李卫芳, 周兵, 间小红. 中国入侵克隆植物入侵性、克隆方式及地理起源. 生物多样性, 2016, 24 (1), 12-19.

http://www.biodiversity-science.net/CN/10.17520/biods.2015190

Xu ZH, Zhu LQ, Yuan XF, Chen WM, Lin YB, Xie GX (2011) Invasion and expansion characteristics of regional adventitious noxious weed Soliva anthemifolia (Juss.) R. Br. and corresponding controlling countermeasures. Ecology and Environmental Sciences, 20, 980985. (in Chinese with English abstract) [徐正浩, 朱丽靑, 袁侠凡, 陈为民, 林云彪, 谢国雄 (2011) 区域性外来恶性杂草裸柱菊的入侵扩散特征及防治对策. 生态环境学报, 20, 980985.]

Zeng JJ, Xiao YA, Sun M (2010) Reproductive traits associated with invasiveness in Coreopsis lanceolata. Chinese Journal of Plant Ecology, 34, 966-972. (in Chinese with English abstract) [曾建军, 肖宜安, 孙敏 (2010) 入侵植物剑叶金鸡菊的繁殖特征及其与入侵性之间的关系. 植物生态学报, 34, 966-972.]

Zhang W, Fan XH, Zhao H (2013) An introduction of the invasive weed Solanum elaeagnifolium. Plant Quarantine, 27, 72-76. (in Chinese with English abstract) [张伟, 范晓红, 赵宏 (2013) 外来 入侵杂草——银毛龙葵. 植物检疫, 27, 72-76.] 